\title{
The Relative Yamabe Invariant
}

\author{
Kazuo AKUtagawa ${ }^{1}$ AND Boris BotvinniK ${ }^{2}$
}

\begin{abstract}
We define the relative Yamabe invariant of a compact smooth manifold with given conformal class on its boundary. In the case of empty boundary the invariant coincides with the Yamabe invariant. We develop approximation techniques which lead to gluing theorems of two manifolds along their boundaries for the relative Yamabe invariant. We show that there are many examples of manifolds with both positive and non-positive relative Yamabe invariants. In particular, we construct families of four-manifolds with strictly negative relative Yamabe invariant and give an exact computation of the invariant.
\end{abstract}

\section{Introduction.}

1.1. General setting. Let $W$ be a compact smooth manifold with boundary $\partial W=M \neq \emptyset$, and $n=\operatorname{dim} W \geq 3$. Let $\operatorname{Riem}(W)$ be the space of all Riemannian metrics on $W$. For a metric $\bar{g} \in \mathcal{R} \operatorname{iem}(W)$ we denote by $H_{\bar{g}}$ the mean curvature along the boundary $\partial W=M$, and $g=\left.\bar{g}\right|_{M}$. We also denote by $[\bar{g}]$ and $[g]$ the corresponding conformal classes, and by $\mathcal{C}(M)$ and $\mathcal{C}(W)$ the space of conformal classes on $M$ and $W$ respectively. Let $\bar{C}$ and $C$ be conformal classes of metrics on $W$ and $M$ respectively. We write $\partial \bar{C}=C$ if $\left.\bar{C}\right|_{M}=C$. Let $\mathcal{C}(W, M)$ be the space of pairs $(\bar{C}, C)$ with $\partial \bar{C}=C$. Denote $\bar{C}^{0}=\left\{\bar{g} \in \bar{C} \mid H_{\bar{g}}=0\right\}$. We call $\bar{C}^{0} \subset \bar{C}$ the normalized conformal class. Let $\mathcal{C}^{0}(W, M)$ be the space of pairs $\left(\bar{C}^{0}, C\right)$ such that $\bar{C}^{0} \subset \bar{C}$ and $(\bar{C}, C) \in \mathcal{C}(W, M)$. It is easy to observe (see [7, formula (1.4)]) that for any conformal class $\bar{C} \in \mathcal{C}(W)$ the subclass $\bar{C}^{0}$ is not empty. Thus there is a natural bijection between the spaces $\mathcal{C}^{0}(W, M)$ and $\mathcal{C}(W, M)$. Let $\bar{g} \in \bar{C}^{0}$ be a metric. Then $\bar{C}^{0}$ could be described as follows:

$$
\bar{C}^{0}=\left\{u^{\frac{4}{n-2}} \bar{g} \mid u \in C_{+}^{\infty}(W) \text { such that } \partial_{\nu} u=0 \text { along } M\right\} .
$$

\footnotetext{
${ }^{1}$ Partially supported by the Grants-in-Aid for Scientific Research (C), Japan Society for the Promotion of Science, No. 11640070.

${ }^{2}$ Partially supported by SFB 478, Münster, and by IHES.
} 
Here $\nu$ is the normal unit (inward) vector field along the boundary, and $C_{+}^{\infty}(W)$ is the space of positive smooth functions on $W$.

1.2. The Einstein-Hilbert functional. Let $C \in \mathcal{C}(M)$ be given. We define the following subspaces of metrics:

$$
\begin{aligned}
& \mathcal{R}_{C}(W, M)=\{\bar{g} \in \mathcal{R i e m}(W) \mid \partial[\bar{g}]=C\} \\
& \mathcal{R}_{C} \operatorname{iem}_{C}^{0}(W, M)=\left\{\bar{g} \in \mathcal{R i e m}_{C}(W) \mid H_{\bar{g}}=0\right\} .
\end{aligned}
$$

We consider the normalized Einstein-Hilbert functional

$$
I: \operatorname{Riem}_{C}^{0}(W, M) \rightarrow \mathbf{R}, \quad I(\bar{g})=\frac{\int_{W} R_{\bar{g}} d \sigma_{\bar{g}}}{\operatorname{Vol}_{\bar{g}}(W)^{\frac{n-2}{n}}},
$$

where $R_{\bar{g}}$ is the scalar curvature and $d \sigma_{\bar{g}}$ is the volume element. As in the case of closed manifolds, we have the following result.

Theorem 1.1. Critical points of the functional I on the space $\operatorname{Riem}_{C}^{0}(W, M)$ coincide with the set of Einstein metrics $\bar{g}$ on $W$ with $\partial[\bar{g}]=C$ and $H_{\bar{g}}=0$.

Remark 1. One can restrict the Einstein-Hilbert functional $I$ on some other subspaces of metrics in $\mathcal{R i e m}(W)$. However, we claim that the subspace $\mathcal{R i e m}_{C}^{0}(W, M)$ is indeed a suitable one for the Einstein-Hilbert functional. For instance, the sets of critical points of the functionals $\left.I\right|_{\mathcal{R i e m}(W)}$ and $\left.I\right|_{\mathcal{R}^{2} \operatorname{mom}_{C}(W, M)}$ are empty. Furthermore, consider the subspace

$$
\mathcal{R i e m}_{C}^{\text {const }}(W, M):=\left\{\bar{g} \in \mathcal{R i e m}_{C}(W, M) \mid d H_{\bar{g}}=c \text { for some constant } c\right\} .
$$

Then the set of critical points of the functional $\left.I\right|_{\mathcal{R i e m}_{C}^{\text {const }}(W, M)}$ is also empty. These facts easily follow from the proof of Theorem 1.1.

1.3. Relative Yamabe invariants. Similarly to the case of closed manifolds, the functional $I$ is not bounded. It is easy to prove that for any manifold $W$ of $\operatorname{dim} W \geq 3$ with $\partial W=M$, and any conformal class $C \in \mathcal{C}(M):$

$$
\inf _{\bar{g} \in \mathcal{R} \operatorname{iem}_{C}^{0}(W, M)} I(\bar{g})=-\infty, \quad \sup _{\bar{g} \in \operatorname{Riem}_{C}^{0}(W, M)} I(\bar{g})=\infty .
$$

Let $(\bar{C}, C) \in \mathcal{C}(W, M)$. We define the relative Yamabe constant of $(\bar{C}, C)$ as

$$
Y_{\bar{C}}(W, M ; C)=\inf _{\bar{g} \in \bar{C}^{0}} I(\bar{g}) .
$$


Remark 2. We notice that the relative Yamabe constant $Y_{\bar{C}}(W, M ; C)$ coincides with the constant $Q(W)$ (up to a universal positive factor depending only on the dimension of $W$ ) defined by J. Escobar [7] for each pair of conformal classes $(\bar{C}, C) \in \mathcal{C}(W, M)$.

The relative Yamabe constant $Y_{\bar{C}}(W, M ; C)$ is related to the Yamabe problem on a compact manifold with boundary which was solved by P. Cherrier [6] and J. Escobar [7] under some restrictions. Indeed, P. Cherrier proved the existence of a minimizer for the Yamabe functional $\left.I\right|_{\bar{C}^{0}}$ provided

$$
Y_{\bar{C}}(W, M ; C)<Y_{\left[\bar{g}_{0}\right]}\left(S_{+}^{n}, S^{n-1} ;\left[g_{0}\right]\right) .
$$

Here $S_{+}^{n}$ is a round hemisphere with the standard metric $\bar{g}_{0}$, and $S^{n-1} \subset S_{+}^{n}$ is the equator with $g_{0}=\left.\bar{g}_{0}\right|_{S^{n-1}}$. More generally, J. Escobar [7] solved the Yamabe problem under the restrictions we list below. The Escobar's result includes the case when the inequality (1.1) is satisfied. Here is the list of conditions given in [7]:

(a) $n=3,4$ or 5 ,

(b) $W$ has a nonumbilic point on $M=\partial W$,

(c) $M$ is umbilic in $W$ and $W$ is locally conformally flat,

(d) $n \geq 6$, and $M$ is umbilic in $W$ and the Weyl tensor $W_{\bar{C}} \not \equiv 0$ on $M$.

Notice that the conditions (1.2) are conformally invariant. We denote

$$
\mathcal{C}^{E s c}(W, M)=\left\{\begin{array}{l|l}
(\bar{C}, C) \in \mathcal{C}(W, M) & \begin{array}{c}
\text { at least one of the conditions } \\
\text { (a)-(d) in (1.2) is satisfied }
\end{array}
\end{array}\right\} .
$$

Remark 3. It is easy to see that $\mathcal{C}^{E s c}(W, M) \subset \mathcal{C}(W, M)$ is open dense.

We state the Escobar's result using the terms introduced above.

Theorem 1.2. ([7, Theorem 6.1]) Let $W$ be a compact manifold with boundary $\partial W=M \neq \emptyset$, and $(\bar{C}, C) \in \mathcal{C}^{E s c}(W, M)$. Then there exists a metric $\check{g} \in \bar{C}^{0}$ such that $Y_{\bar{C}}(W, M ; C)=I(\check{g})$. Such metric $\check{g}$ is called a relative Yamabe metric.

Remark 4. A relative Yamabe metric $\check{g} \in \bar{C}^{0}$ has constant scalar curvature $R_{\breve{g}}=Y_{\bar{C}}(W, M ; C) \cdot \operatorname{Vol}_{\breve{g}}(W)^{-\frac{2}{n}}$.

We define the relative Yamabe invariants $Y(W, M, C)$ and $Y(W, M)$ (see [13], [25] for the Yamabe invariant of a closed manifold):

$$
Y(W, M, C)=\sup _{\bar{C}, \partial \bar{C}=C} Y_{\bar{C}}(W, M ; C), \quad Y(W, M)=\sup _{C \in \mathcal{C}(M)} Y(W, M ; C) .
$$


The invariant $Y(W, M ; C)$ has clear geometric meaning in terms of positive scalar curvature (abbreviated as psc). We call a conformal class $C \in \mathcal{C}(M)$ positive if the Yamabe constant $Y_{C}(M)>0$. Of course, it means that the conformal class $C$ contains a psc-metric. The following statement follows from the above definitions.

Claim 1.3. (1) Let $C \in \mathcal{C}(M)$ be a positive conformal class. Then $Y(W, M ; C)>0$ if and only if any psc-metric $g \in C$ can be extended conformally to a psc-metric $\bar{g}$ on $W$ with $H_{\bar{g}}=0$ along $M$.

(2) The invariant $Y(W, M)$ is a diffeomorphism invariant. Furthermore, $Y(W, M)>0$ if and and only if there exists a psc-metric $\bar{g}$ on $W$ with $H_{\bar{g}}=0$ along $M$.

We present our main results on the relative Yamabe invariants in the next section.

1.4. Acknowledgments. Both authors would like to acknowledge partial financial support provided by the Department of Mathematics at the University of Oregon, SFB 478 - Geometrische Strukturen in der Mathematik and by the Grants-in-Aid for Scientific Research, Japan. We would like also to thank Mitsuhiro Itoh and Thomas Schick for useful discussions and their hospitality while we visited the University of Tsukuba and during our visit at Münster. The second author would like to thank Michael Joachim, Wolfgang Lück, and the members of the geometry-topology in Münster for very interesting mathematical discussions. This work was also partially supported by IHES, and the second author would like to thank IHES for hospitality. Both authors are grateful to the referee for valuable remarks, and to Masashi Ishida for useful discussions.

\section{Overview of the results.}

\subsection{Minimal boundary condition and approximation theorems.} First, one can notice that the minimal boundary condition $H_{\bar{g}}=0$ is rather weak for applications. For instance, to apply the Atiyah-Patodi-Singer index theory, one needs much stronger condition that a metric $\bar{g}$ is a product metric near the boundary. The closest geometric approximation to a product metric near the boundary is when this boundary is totally geodesic. In more detail, let $\bar{g} \in \mathcal{R i e m}_{C}^{0}(W, M), g=\left.\bar{g}\right|_{M}$. Clearly any metric from the normalized conformal class $[\bar{g}]^{0}$ is totally geodesic on $M$ if $\bar{g}$ is. We call the conformal class $[\bar{g}]$ of such metric $\bar{g}$ umbilic. We denote by $\mathcal{C}_{C}^{u m}(W, M) \subset \mathcal{C}_{C}(W, M):=$ $\{\bar{C} \in \mathcal{C}(W) \mid \partial \bar{C}=C\}$ the subspace of umbilic conformal classes. 
Our first aim is to prove a generalization (Proposition 4.5) of the approximation theorem due to Kobayashi [13]. We show that any metric $\bar{g}$ with totally geodesic boundary is $C^{1}$-close to a metric $\tilde{g}$ which is conformally equivalent to a product metric near the boundary. Moreover, we show that the scalar curvature $R_{\bar{g}}$ is $C^{0}$-close to $R_{\tilde{g}}$ of $\tilde{g}$.

Next, we prove the approximation Theorem 4.6 under the minimal boundary condition. Theorem 4.6 gives us a fundamental tool on the relative Yamabe invariants. In particular, we prove the following result.

Theorem 2.1. For any $\bar{C} \in \mathcal{C}_{C}(W, M), \bar{g} \in \bar{C}$ and any $\varepsilon>0$ there exist $a$ conformal class $\tilde{C} \in \mathcal{C}_{C}^{u m}(W, M)$ and a metric $\tilde{g} \in \tilde{C}^{0}$ such that

$$
\left\{\begin{array}{l}
\tilde{C} \text { and } \bar{C} \text { are } C^{0} \text {-close conformal classes, } \\
\left|Y_{\tilde{C}}(W, M ; C)-Y_{\bar{C}}(W, M ; C)\right|<\varepsilon \\
\tilde{g} \sim g+d r^{2} \quad(\text { conformally equivalent near } M),
\end{array}\right.
$$

where $g=\left.\bar{g}\right|_{M}$. More precisely,

$$
\begin{aligned}
& \tilde{g}=\left(1+\frac{r^{2}}{2} f(x)\right)^{\frac{4}{n-2}}\left(g+d r^{2}\right) \quad \text { near } M, \text { where } \\
& f(x)=-\frac{n-2}{4(n-1)}\left(\left.R_{\bar{g}}\right|_{M}-R_{g}\right) \quad \text { on } M .
\end{aligned}
$$

We define the "umbilic Yamabe invariant" $Y^{u m}(W, M ; C)$ as

$$
Y^{u m}(W, M ; C)=\sup _{\tilde{C} \in \mathcal{C}_{C}^{u m}(W, M)} Y_{\tilde{C}}(W, M ; C) .
$$

Theorem 2.1 leads to the following conclusion.

Corollary 2.2. $Y^{u m}(W, M ; C)=Y(W, M ; C)$.

2.2. Gluing Theorem. We analyze a gluing procedure for manifolds equipped with conformal structures. Let $W_{1}, W_{2}$ be two compact manifolds of $\operatorname{dim} W_{1}=\operatorname{dim} W_{2} \geq 3$ with boundaries

$$
\partial W_{1}=M_{1}=M_{0} \sqcup M, \quad \text { and } \quad \partial W_{2}=M_{2}=M_{0} \sqcup M^{\prime}
$$

endowed with conformal classes $C_{1}=C_{0} \sqcup C \in \mathcal{C}\left(M_{1}\right), C_{2}=C_{0} \sqcup C^{\prime} \in \mathcal{C}\left(M_{2}\right)$, where $C_{0} \in \mathcal{C}\left(M_{0}\right), C \in \mathcal{C}(M), C^{\prime} \in \mathcal{C}\left(M^{\prime}\right)$. Let $W=W_{1} \cup_{M_{0}}\left(-W_{2}\right)$ be the union of $W_{1}$ and $W_{2}$ along common boundary $M_{0}$.

We study the case when the conformal class $C_{0} \in \mathcal{C}\left(M_{0}\right)$ is positive, and the relative Yamabe invariants $Y\left(W_{j}, M_{j} ; C_{j}\right), j=1,2$, are positive as well. We essentially use the approximation Theorem 4.6 to prove the following result (Theorem 5.1): 
Theorem 2.3. Let $C_{0} \in \mathcal{C}\left(M_{0}\right)$ be a positive conformal class, and $Y\left(W_{j}, M_{j} ; C_{j}\right)>0$ for $j=1,2$. Then $Y\left(W, \partial W ; C \sqcup C^{\prime}\right)>0$.

Thus new examples of manifolds with positive Yamabe invariant may be easily constructed by gluing two manifolds with positive relative Yamabe invariant.

2.3. Yamabe invariant of the double. Let $W$ be a compact $n$ manifold with boundary $\partial W=M \neq \emptyset$, and $M=M_{0} \sqcup M_{1}$. (Here $M_{1}$ may be empty.) Let $\left(\bar{C}, C=C_{0} \sqcup C_{1}\right)$ be a pair of conformal classes on $(W, M)$, where $C_{0} \in \mathcal{C}\left(M_{0}\right), C_{1} \in \mathcal{C}\left(M_{1}\right)$. Let $X=W \cup_{M_{0}}(-W)$ be the double of $W$ along $M_{0}$. Then, the boundary of $X$ is $\partial X=M_{1} \sqcup\left(-M_{1}\right)$. We prove the following result (Theorem 5.3).

Theorem 2.4. Let $W, X$ be manifolds as above, and $C=C_{0} \sqcup C_{1} \in \mathcal{C}(\partial W)$, where $C_{0} \in \mathcal{C}\left(M_{0}\right), C_{1} \in \mathcal{C}\left(M_{1}\right)$. Then

(1) $Y\left(X, M_{1} \sqcup\left(-M_{1}\right) ; C_{1} \sqcup C_{1}\right) \geq \begin{cases}Y(W, M ; C) & \text { if } Y(W, M ; C)>0, \\ 2^{\frac{2}{n}} Y(W, M ; C) & \text { if } Y(W, M ; C) \leq 0 .\end{cases}$

$$
Y\left(X, M_{1} \sqcup\left(-M_{1}\right)\right) \geq \begin{cases}Y(W, M) & \text { if } Y(W, M)>0, \\ 2^{\frac{2}{n}} Y(W, M) & \text { if } Y(W, M) \leq 0 .\end{cases}
$$

When the manifolds $\partial W=M=M_{0}$, and $M_{1}$ is empty (in this case, the boundary of $X=W \cup_{M}(-W)$ is empty), the following holds (Corollary 5.4):

Corollary 2.5. Let $\partial W=M$, and let $X=W \cup_{M}(-W)$ be the double of $W, \partial X=\emptyset$. Then

$$
Y(X) \geq \begin{cases}Y(W, M) & \text { if } Y(W, M)>0 \\ 2^{\frac{2}{n}} Y(W, M) & \text { if } Y(W, M) \leq 0 .\end{cases}
$$

In particular, if $Y(X) \leq 0$, then $Y(W, M) \leq 2^{-\frac{2}{n}} Y(X) \leq 0$.

We use these results to give examples of manifolds with non-positive Yamabe invariant.

Corollary 2.6. Let $N$ be an enlargeable closed manifold. Then

$$
2^{\frac{2}{n}} Y\left(N \backslash \operatorname{int}\left(D^{n}\right), S^{n-1}\right) \leq Y(N \#(-N)) \leq 0 .
$$

In particular, $Y\left(N \backslash \operatorname{int}\left(D^{n}\right), S^{n-1}\right) \leq 0$. 
2.4. Four-manifolds with negative relative Yamabe invariant. Let $X$ be a minimal closed symplectic 4-manifold of general type with an involution $\iota$. Assume that the fixed point set $\operatorname{Fix}(\iota)$ of $\iota$ is an oriented closed 3-manifold $M$ (possibly disconnected). We notice that in this situation the manifold $X=W \cup_{M}(-W)$ is the double of the closure $W$ of a connected component of $X \backslash M$ (Lemma 6.2). We use our technique combining with the estimates given in [16], [21] (cf. [27]) to prove that $Y\left(W \# \ell\left(S^{1} \times S^{3}\right), M\right)<0$, see Theorem 6.3. To evaluate the relative Yamabe invariant, we specify this construction as follows.

Let $N$ be a product $\Sigma_{k_{1}} \times \Sigma_{k_{2}}$ of closed Riemann surfaces of genus $k_{1}, k_{2} \geq$ 2, and $\bar{g}$ the product metric $g_{1} \times g_{2}$ of $g_{1}$ on $\Sigma_{k_{1}}$ and $g_{2}$ on $\Sigma_{k_{2}}$, respectively, with constant negative curvature -1 . Let $\phi:\left(\Sigma_{k_{1}}, g_{1}\right) \rightarrow\left(\Sigma_{k_{1}}, g_{1}\right)$ be an involutive isometry such that $\operatorname{Fix}(\phi)$ consists of $k_{1}+1$ disjoint simple closed geodesics (such $\phi$ always exists for an appropriate $g_{1}$ if $k_{1} \geq 2$ ). Then the map $\iota:=\phi \times I d: N=\Sigma_{k_{1}} \times \Sigma_{k_{2}} \rightarrow N$ is also an involutive isometry with $\operatorname{Fix}(\iota)=\operatorname{Fix}(\phi) \times \Sigma_{k_{2}}$ consisting of $k_{1}+1$ disjoint closed totally geodesic 3 -submanifolds. Then it follows that $N=W \cup_{\partial W}(-W)$, where $W$ is the closure of a connected components of $N \backslash \operatorname{Fix}(\iota)$. We obtain the following result (see Corollary 6.4).

Corollary 2.7. For all $\ell \geq 0$ the relative Yamabe invariant of the manifold $W \# \ell\left(S^{1} \times S^{3}\right)$ is given by

$$
Y\left(W \# \ell\left(S^{1} \times S^{3}\right), \partial W\right)=\frac{1}{\sqrt{2}} Y(N)=-8 \sqrt{2} \pi \sqrt{\left(k_{1}-1\right)\left(k_{2}-1\right)}<0 .
$$

The rest of the paper is organized as follows. We prove Theorem 1.1 in Section 3. Then we prove the approximation theorems in Section 4. We give a gluing construction in Section 5. In Section 6, we analyze the Yamabe invariant for a double and give examples of manifolds with non-positive or negative relative Yamabe invariant. In the last Section 7 we define and study the moduli space of positive conformal classes and introduce the notions of conformal concordance and conformal cobordism.

\section{Proof of Theorem 1.1.}

Let $\bar{g} \in \mathcal{R i e m}_{C}(W, M)$ be a metric and $\{\bar{g}(t)\}$ a smooth variation of $\bar{g}$ in the space $\mathcal{R i e m}_{C}(W, M)$, where $\bar{g}(0)=\bar{g}$. We consider first a general variation, i.e. $\{\bar{g}(t)\}$ is not necessarily contained in the subspace $\operatorname{Riem}_{C}^{0}(W, M) \subset$ $\operatorname{Riem}_{C}(W, M)$. Now we need the following notations. Let $h=\left.\frac{d}{d t}\right|_{t=0} \bar{g}(t)$ be a variational vector and $g(t)=\left.\bar{g}(t)\right|_{M}$, where $g(0)=g$. 
Remark 5. We observe that the condition $g(t) \in C$ implies that $h=f g$ on $M$, where $f \in C^{\infty}(M)$.

Let $r=r(t)$ be the distance function to the boundary $M$ in $W$ with respect to the metric $\bar{g}(t)$. Let $\nu=\frac{\partial}{\partial r}$ be the unit normal (inward) vector field along the boundary $\partial W=M$. Let $p \in M$, and $\left\{r, x^{1}, \ldots, x^{n-1}\right\}$ be a Fermi coordinate system near $p$. We use indices $\alpha, \beta=0,1, \ldots, n-1$, where 0 corresponds to the normal direction, and $i, j, k=1, \ldots, n-1$ are indices corresponding to the tangent directions (only on the boundary $\partial W=M$ ). We denote by $(\cdot)^{\prime}=\left.\frac{d}{d t}(\cdot)\right|_{t=0}$ the variational derivative evaluated at $t=0$. In order to prove Theorem 1.1, it is enough to prove the following formula.

Claim 3.1. Let $\{\bar{g}(t)\}$ be a variation as above. Then

$$
\left(\int_{W} R_{\bar{g}(t)} d \sigma_{\bar{g}(t)}\right)^{\prime}=-\int_{W}\left\langle\operatorname{Ric}_{\bar{g}}-\frac{1}{2} R_{\bar{g}} \bar{g}, h\right\rangle_{\bar{g}} d \sigma_{\bar{g}}-\int_{M}\left(2 H_{\bar{g}}^{\prime}+f H_{\bar{g}}\right) d \sigma_{g},
$$

where $\operatorname{Ric}_{\bar{g}}$ is the Ricci curvature of $\bar{g}$.

Proof. We denote by $\bar{\nabla}$ and $\nabla$ corresponding Levi-Civita connections with respect to the metrics $\bar{g}$ and $g$. Standard calculation gives:

$$
\begin{aligned}
\left(R_{\bar{g}(t)}\right)^{\prime} & =-\bar{\nabla}^{\alpha} \bar{\nabla}_{\alpha}\left(\operatorname{Tr}_{\bar{g}} h\right)+\bar{\nabla}^{\alpha} \bar{\nabla}^{\beta} h_{\alpha \beta}-\left\langle\operatorname{Ric}_{\bar{g}}, h\right\rangle_{\bar{g}}, \\
\left(d \sigma_{\bar{g}(t)}\right)^{\prime} & =\left\langle\frac{1}{2} R_{\bar{g}} \bar{g}, h\right\rangle_{\bar{g}} d \sigma_{\bar{g}} .
\end{aligned}
$$

The formula (3.1) together with Gauss' divergence formula gives

$$
\begin{aligned}
\left(\int_{W} R_{\bar{g}(t)} d \sigma_{\bar{g}(t)}\right)^{\prime}= & -\int_{W}\left\langle\operatorname{Ric}_{\bar{g}}-\frac{1}{2} R_{\bar{g}} \bar{g}, h\right\rangle_{\bar{g}} d \sigma_{\bar{g}} \\
& +\int_{M}\left\langle\bar{\nabla}\left(\operatorname{Tr}_{\bar{g}} h\right), \nu\right\rangle_{\bar{g}} d \sigma_{g}-\int_{M} \sum_{\alpha=0}^{n-1}\left(\bar{\nabla}_{e_{\alpha}} h\right)\left(e_{\alpha}, \nu\right) d \sigma_{g} .
\end{aligned}
$$

Here $\left\{e_{\alpha}\right\}=\left\{\nu, e_{1}, \ldots, e_{n-1}\right\}$ is a local orthonormal field. We denote

$$
B_{I}=\left\langle\bar{\nabla}\left(\operatorname{Tr}_{\bar{g}} h\right), \nu\right\rangle_{\bar{g}}, \quad B_{I I}=-\sum_{\alpha=0}^{n-1}\left(\bar{\nabla}_{e_{\alpha}} h\right)\left(e_{\alpha}, \nu\right) .
$$

Let $p \in M$ be an arbitrary point of the boundary. As before, let $\nu$ be the unit vector field normal (inward) to the boundary such that $\bar{\nabla}_{\nu} \nu=0$, and 
let $\left\{e_{i}\right\}$ be an orthonormal frame near $p$ in $W$ such that $\nabla_{e_{i}} e_{j}=0$ at $p$ and $t=0$. We notice that, in general, $\bar{\nabla}_{e_{i}} e_{j}$ does not vanish at $p$. We have the second fundamental form of $M$ :

$$
A_{i j}=A\left(e_{i}, e_{j}\right)=\bar{g}\left(\bar{\nabla}_{e_{i}} e_{j}, \nu\right)=-\bar{g}\left(\nabla_{e_{i}} \nu, e_{j}\right) .
$$

Then we have $H=g^{i j} A_{i j}$ the mean curvature of the boundary $M$. We have:

$$
H=-\sum_{i=0}^{n-1} \bar{g}\left(\bar{\nabla}_{e_{i}} \nu, e_{i}\right)=\sum_{\alpha=0}^{n-1} \bar{g}\left(\bar{\nabla}_{e_{\alpha}} \nu, e_{\alpha}\right)=-\nabla_{\alpha} \nu^{\alpha}=-\left(\partial_{\alpha} \nu^{\alpha}+\bar{\Gamma}_{\alpha \beta}^{\alpha} \nu^{\beta}\right)
$$

Here $\left\{x^{\alpha}\right\}=\left\{r, x^{1}, \ldots, x^{n-1}\right\}$ is a Fermi coordinate system near $p$ in $W$, and $\partial_{\alpha}=\frac{\partial}{\partial x^{\alpha}}\left(\right.$ and $\partial_{\alpha}=e_{\alpha}$ at $\left.p\right)$. We have:

$$
\begin{aligned}
H^{\prime} & =-\left[\partial_{\alpha}\left(\nu^{\prime}\right)^{\alpha}+\bar{\Gamma}_{\alpha \beta}^{\alpha}\left(\nu^{\prime}\right)^{\beta}+\left(\bar{\Gamma}^{\prime}\right)_{\alpha \beta}^{\alpha} \nu^{\beta}\right] \\
& =-\left[\bar{\nabla}_{\alpha}\left(\nu^{\prime}\right)^{\alpha}+\frac{1}{2}\left(\bar{\nabla}_{\alpha} h_{\beta}^{\alpha}+\bar{\nabla}_{\beta} h_{\alpha}^{\alpha}-\bar{\nabla}^{\alpha} h_{\alpha \beta}\right) \nu^{\beta}\right] \\
& =-\bar{\nabla}_{\alpha}\left(\nu^{\prime}\right)^{\alpha}-\frac{1}{2} \bar{\nabla}_{\beta}\left(\operatorname{Tr}_{\bar{g}} h\right) \nu^{\beta}=-\bar{\nabla}_{\alpha}\left(\nu^{\prime}\right)^{\alpha}-\frac{1}{2}\left\langle\bar{\nabla}\left(\operatorname{Tr}_{\bar{g}} h\right), \nu\right\rangle_{\bar{g}}
\end{aligned}
$$

Thus we obtain

$$
B_{I}=\left\langle\bar{\nabla}\left(\operatorname{Tr}_{\bar{g}} h\right), \nu\right\rangle_{\bar{g}}=-\left(2 H^{\prime}+2 \bar{\nabla}_{\alpha}\left(\nu^{\prime}\right)^{\alpha}\right) .
$$

Now we compute the term $B_{I I}$. We have

$$
\begin{aligned}
\sum_{\alpha=0}^{n-1}\left(\bar{\nabla}_{e_{\alpha}} h\right)\left(e_{\alpha}, \nu\right) & =\sum_{\alpha=0}^{n-1}\left(\bar{\nabla}_{e_{\alpha}} h\left(e_{\alpha}, \nu\right)-h\left(\bar{\nabla}_{e_{\alpha}} e_{\alpha}, \nu\right)-h\left(e_{\alpha}, \bar{\nabla}_{e_{\alpha}} \nu\right)\right) \\
& =\bar{\nabla}_{\nu} h(\nu, \nu)+\sum_{i=1}^{n-1} \bar{\nabla}_{e_{i}} h\left(e_{i}, \nu\right)-h_{00} H+h^{i k} A_{i k}
\end{aligned}
$$

since

$$
\begin{aligned}
& \sum_{\alpha=0}^{n-1} \bar{\nabla}_{e_{\alpha}} \nu=\bar{\nabla}_{\nu} \nu+\sum_{i=1}^{n-1} \bar{\nabla}_{e_{i}} \nu, \quad \bar{\nabla}_{\nu} \nu=0, \text { and } \\
& \bar{\nabla}_{e_{i}} \nu=-\sum_{k=1}^{n-1} A_{i k} e_{k}, \quad \sum_{\alpha=0}^{n-1} \bar{\nabla}_{e_{\alpha}} e_{\alpha}=\bar{\nabla}_{\nu} \nu+\sum_{i=1}^{n-1} \bar{\nabla}_{e_{i}} e_{i}=H \nu
\end{aligned}
$$


Notice that $h^{i k}=f g^{i k}$ and $\bar{\nabla}_{e_{i}} h\left(e_{j}, \nu\right)=\nabla_{e_{i}} h\left(e_{j}, \nu\right)$. Thus we have

$$
B_{I I}=-\sum_{\alpha=0}^{n-1}\left(\bar{\nabla}_{e_{\alpha}} h\right)\left(e_{\alpha}, \nu\right)=-\bar{\nabla}_{\nu} h(\nu, \nu)-\sum_{i=1}^{n-1} \nabla_{e_{i}} h\left(e_{i}, \nu\right)+h_{00} H-f H .
$$

To continue, we notice that $\bar{g}(\nu, \nu)=1$ implies

$$
0=\bar{g}^{\prime}(\nu, \nu)+2 \bar{g}\left(\nu^{\prime}, \nu\right)=h(\nu, \nu)+2 \bar{g}\left(\nu^{\prime}, \nu\right) .
$$

Also we have

$$
0=\bar{\nabla}_{\nu} h(\nu, \nu)+2 \bar{g}\left(\bar{\nabla}_{\nu} \nu^{\prime}, \nu\right)+2 \bar{g}\left(\nu^{\prime}, \bar{\nabla}_{\nu} \nu\right)=\bar{\nabla}_{\nu} h(\nu, \nu)+2 \bar{g}\left(\bar{\nabla}_{\nu} \nu^{\prime}, \nu\right)
$$

since $\bar{\nabla}_{\nu} \nu=0$. Thus we have that

$$
2 \bar{g}\left(\bar{\nabla}_{\nu} \nu^{\prime}, \nu\right)=-\bar{\nabla}_{\nu} h(\nu, \nu) .
$$

Then the identity $\bar{g}\left(\nu, e_{i}\right)=0$ implies

$$
0=\bar{g}^{\prime}\left(\nu, e_{i}\right)+\bar{g}\left(\nu^{\prime}, e_{i}\right)+\bar{g}\left(\nu, e_{i}^{\prime}\right) .
$$

Notice that $e_{i}^{\prime} \in T_{p} M$ since $g(t) \in C$. Thus

$$
0=\bar{g}^{\prime}\left(\nu, e_{i}\right)+\bar{g}\left(\nu^{\prime}, e_{i}\right)
$$

Now it follows that

$$
0=\sum_{i=1}^{n-1}\left(\bar{\nabla}_{e_{i}} h\left(\nu, e_{i}\right)+\bar{g}\left(\bar{\nabla}_{e_{i}} \nu^{\prime}, e_{i}\right)+\bar{g}\left(\nu^{\prime}, \bar{\nabla}_{e_{i}} e_{i}\right)\right) .
$$

Notice that $\sum_{i=1}^{n-1} \bar{\nabla}_{e_{i}} e_{i}=H \nu$, and $H \bar{g}\left(\nu^{\prime}, \nu\right)=-\frac{1}{2} h(\nu, \nu)$. Thus we obtain

$$
2 \sum_{i=1}^{n-1} \bar{g}\left(\bar{\nabla}_{e_{i}} \nu^{\prime}, e_{i}\right)=-2 \sum_{i=1}^{n-1} \nabla_{e_{i}} h\left(\nu, e_{i}\right)+h_{00} H .
$$

We combine (3.4) and (3.5) to obtain

$$
\begin{aligned}
2 \bar{\nabla}_{\alpha}\left(\nu^{\prime}\right)^{\alpha} & =2 \sum_{\alpha=0}^{n-1} \bar{g}\left(\bar{\nabla}_{e_{\alpha}} \nu^{\prime}, e_{\alpha}\right)=2\left[\bar{g}\left(\bar{\nabla}_{\nu} \nu^{\prime}, \nu\right)+\sum_{i=1}^{n-1} \bar{g}\left(\bar{\nabla}_{e_{i}} \nu^{\prime}, e_{i}\right)\right] \\
& =-\bar{\nabla}_{\nu} h(\nu, \nu)-2 \sum_{i=1}^{n-1} \nabla_{e_{i}} h\left(\nu, e_{i}\right)+h_{00} H .
\end{aligned}
$$


Now it follows from (3.2), (3.3) and (3.6) that

$$
\begin{aligned}
B_{I}+B_{I I} & =-2 H^{\prime}-2 \bar{\nabla}_{\alpha}\left(\nu^{\prime}\right)^{\alpha}-\bar{\nabla}_{\nu} h(\nu, \nu)-\sum_{i=1}^{n-1} \nabla_{e_{i}} h\left(e_{i}, \nu\right)+h_{00} H-f H \\
& =-2 H^{\prime}-\sum_{i=1}^{n-1} \nabla_{e_{i}} h\left(\nu, e_{i}\right)-f H .
\end{aligned}
$$

Denote $\theta(v)=h(\nu, v)$ for $v \in T_{x} M$, so $\theta$ is a 1-form on $M$. We notice that

$$
\nabla_{e_{j}} \theta\left(e_{i}\right)=\left(\nabla_{e_{i}} \theta\right)\left(e_{j}\right)+\theta\left(\nabla_{e_{i}} e_{j}\right)=\left(\nabla_{e_{i}} \theta\right)\left(e_{j}\right) \text { since } \nabla_{e_{i}} e_{j}=0 \text { at } p \text {. }
$$

Thus we have that

$$
B_{I}+B_{I I}=-2 H^{\prime}-f H-\nabla_{i} \theta^{i} \quad \text { on } M .
$$

This proves Claim 3.1 and concludes the proof of Theorem 1.1.

\section{Approximation Theorems.}

4.1. Kobayashi approximation lemma. First we reformulate several known facts in our terms. The following fact follows from a modification of the continuity property of the Yamabe constant due to Bérard Bergery.

Lemma 4.1. (cf. [3, Proposition 4.31]) Let $\bar{g}_{i}, \bar{g} \in \mathcal{R i e m}_{C}^{0}(W, M)$ be Riemannian metrics, and $\bar{C}_{i}=\left[\bar{g}_{i}\right], \bar{C}=[\bar{g}]$. Assume that

$$
\begin{cases}\bar{g}_{i} \rightarrow \bar{g} & \text { in the } C^{0} \text {-topology on } W, \text { and } \\ R_{\bar{g}_{i}} \rightarrow R_{\bar{g}} & \text { in the } C^{0} \text {-topology on } W .\end{cases}
$$

Then $Y_{\bar{C}_{i}}(W, M, C) \rightarrow Y_{\bar{C}}(W, M, C)$.

Now we recall the results due to O. Kobayashi [13].

Lemma 4.2. (O. Kobayashi [13]) For any $\delta>0$ there exist a smooth nonnegative function $w_{\delta}$ and a positive constant $\varepsilon(\delta)(0<\varepsilon(\delta)<\delta)$ such that

(i) $\begin{cases}w_{\delta}(t) \equiv 1 & \text { on }[0, \varepsilon(\delta)], \\ w_{\delta}(t) \equiv 0 & \text { on }[\delta, \infty),\end{cases}$

(ii) $\left|t \dot{w}_{\delta}(t)\right|<\delta$ for $t \geq 0$,

(iii) $\left|t^{2} \ddot{w}_{\delta}(t)\right|<\delta$ for $t \geq 0$.

(see Fig. 4.1.)

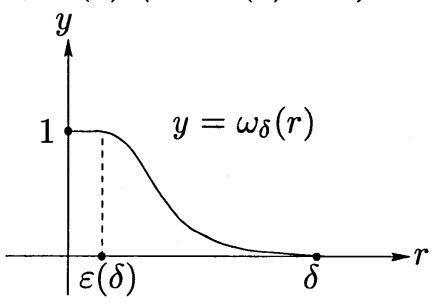

Fig. 4.1 
Lemma 4.3. (O. Kobayashi [13]) Let $\bar{g}, \tilde{g} \in \mathcal{R i e m}(W)$, and $h=\tilde{g}-\bar{g}$. Then

$$
\begin{aligned}
& R_{\tilde{g}}-R_{\bar{g}}=P_{\bar{g}}(h)+Q_{\bar{g}}(h), \text { where } \\
& \left\{\begin{array}{l}
P_{\bar{g}}(h)=-\Delta_{\bar{g}}\left(\operatorname{Tr}_{\bar{g}} h\right)+\bar{\nabla}^{i} \bar{\nabla}^{j} h_{i j}-\left\langle h, \operatorname{Ric}_{\bar{g}}\right\rangle_{\bar{g}}, \\
\left|Q_{\bar{g}}(h)\right| \leq C\left(|\bar{\nabla} h|^{2} q^{3}+|h| \cdot\left|\bar{\nabla}^{2} h\right| q^{2}+\left(|h| \cdot\left|\bar{\nabla}^{2} h\right|+\left|\operatorname{Ric}_{\bar{g}}\right| \cdot|h|^{2}\right) q\right),
\end{array}\right.
\end{aligned}
$$

where the constant $C>0$ depends only on $n=\operatorname{dim} W$, and $q \in C_{+}^{\infty}(W)$ is a function satisfying $q \cdot \tilde{g} \geq \bar{g}$.

Proposition 4.4. Let $W$ be a compact manifold with boundary $\partial W=M$, and let metrics $\bar{g}, \tilde{g} \in \mathcal{R i e m}_{C}(W, M)$ such that $j_{M}^{1} \bar{g}=j_{M}^{1} \tilde{g}$ (i.e. $\bar{g}$ coincides with $\tilde{g}$ up to their first derivatives on $M$ ), and $R_{\bar{g}}=R_{\tilde{g}}$ on $M$. Then the family of metrics

$$
\tilde{g}_{\delta}=\bar{g}+w_{\delta}(r)(\tilde{g}-\bar{g}) \in \mathcal{R i e m}_{C}(W, M)
$$

satisfies the following properties:

(i) $\tilde{g}_{\delta} \rightarrow \bar{g}$ in the $C^{1}$-topology on $W($ as $\delta \rightarrow 0)$,

(ii) $R_{\tilde{g}_{\delta}} \rightarrow R_{\bar{g}}$ in the $C^{0}$-topology on $W($ as $\delta \rightarrow 0)$,

(iii) $\tilde{g}_{\delta} \equiv \tilde{g}$ on the collar $U_{\varepsilon(\delta)}(M, \bar{g})=\left\{x \in W \mid \operatorname{dist}_{\bar{g}}(x, M)<\varepsilon(\delta)\right\}$,

(iv) $\tilde{g}_{\delta} \equiv \bar{g}$ on $W \backslash U_{\delta}(M, \bar{g})$.

Proof. The statements (iii), (iv) are obvious. We prove (i) and (ii).

(i) The function $w_{\delta}$ satisfies $\operatorname{supp}\left(w_{\delta}\right) \subset[0, \delta]$. Then it follows

$$
\tilde{g}_{\delta}-\bar{g}=w_{\delta}(r)(\tilde{g}-\bar{g})=O\left(r^{2}\right),
$$

thus $\tilde{g}_{\delta} \rightarrow \bar{g}$ in the $C^{0}$-topology on $W$. Furthermore,

$$
\partial\left(\tilde{g}_{\delta}-\bar{g}\right)=\dot{w}_{\delta}(r)\left(\tilde{g}_{\delta}-\bar{g}\right)+w_{\delta}(r) \partial(\tilde{g}-\bar{g}) .
$$

By the condition on the metrics $\tilde{g}_{\delta}, \bar{g}$,

$$
\tilde{g}_{\delta}-\bar{g}=O\left(r^{2}\right), \quad \partial\left(\tilde{g}_{\delta}-\bar{g}\right)=O(r) .
$$

We use Lemma 4.2 to estimate

$$
\left|\partial \tilde{g}_{\delta}-\partial \bar{g}\right| \leq\left|\dot{w}_{\delta}(r) r\right| \cdot \frac{O\left(r^{2}\right)}{r}+w_{\delta}(r) \cdot O(r) \leq \delta O(\delta)+O(\delta) .
$$


Thus $\partial \tilde{g}_{\delta} \rightarrow \partial \bar{g}$ in the $C^{0}$-topology, and hence $\tilde{g}_{\delta} \rightarrow \bar{g}$ in the $C^{1}$-topology on $W$.

(ii) We use Lemma 4.3 to write

$$
\left\{\begin{aligned}
R_{\tilde{g}_{\delta}}-R_{\bar{g}} & =P_{\bar{g}}\left(w_{\delta}(r)\left(\tilde{g}_{\delta}-\bar{g}\right)\right)+Q_{\bar{g}}\left(w_{\delta}(r)\left(\tilde{g}_{\delta}-\bar{g}\right)\right), \\
w_{\delta}(r)\left(R_{\tilde{g}_{\delta}}-R_{\bar{g}}\right) & =w_{\delta}(r) P_{\bar{g}}\left(\tilde{g}_{\delta}-\bar{g}\right)+w_{\delta}(r) Q_{\bar{g}}\left(\tilde{g}_{\delta}-\bar{g}\right) .
\end{aligned}\right.
$$

We use again Lemma 4.3:

$$
\begin{aligned}
& \left|P_{\bar{g}}\left(w_{\delta}(r)\left(\tilde{g}_{\delta}-\bar{g}\right)\right)-w_{\delta}(r) P_{\bar{g}}\left(\tilde{g}_{\delta}-\bar{g}\right)\right| \\
& \leq C\left(\left|\ddot{w}_{\delta}(r)\right| \cdot\left|w_{\delta}(r)\right| \cdot\left|\tilde{g}_{\delta}-\bar{g}\right|+\left|\dot{w}_{\delta}(r)\right|^{2} \cdot\left|\tilde{g}_{\delta}-\bar{g}\right|+\left|\dot{w}_{\delta}(r)\right| \cdot\left|w_{\delta}(r)\right| \cdot\left|\partial\left(\tilde{g}_{\delta}-\bar{g}\right)\right|\right) \\
& \quad \leq C\left(\left(\left|\ddot{w}_{\delta}(r) r^{2}\right|+\left|\dot{w}_{\delta}(r) r\right|^{2}\right) \frac{O\left(r^{2}\right)}{r^{2}}+\left|\dot{w}_{\delta}(r) r\right| \cdot \frac{O(r)}{r}\right) \leq C_{1} \delta .
\end{aligned}
$$

Similarly we obtain

$$
\left\{\begin{array}{l}
\left|Q_{\bar{g}}\left(w_{\delta}(r)\left(\tilde{g}_{\delta}-\bar{g}\right)\right)\right| \leq C_{2} \delta \\
\left.\mid w_{\delta}(r) Q_{\bar{g}}\left(\tilde{g}_{\delta}-\bar{g}\right)\right) \mid \leq C_{3} \delta
\end{array}\right.
$$

Notice that $\left|w_{\delta}(r)\left(R_{\tilde{g}_{\delta}}-R_{\bar{g}}\right)\right| \leq C_{4} \delta$ since $R_{\tilde{g}} \equiv R_{\bar{g}}$ on $M$. Thus we obtain:

$$
\begin{aligned}
\left|R_{\tilde{g}_{\delta}}-R_{\bar{g}}\right| \leq & \left|P_{\bar{g}}\left(w_{\delta}(r)\left(\tilde{g}_{\delta}-\bar{g}\right)\right)-w_{\delta}(r) P_{\bar{g}}\left(\tilde{g}_{\delta}-\bar{g}\right)\right|+ \\
& \left.\left|Q_{\bar{g}}\left(w_{\delta}(r)\left(\tilde{g}_{\delta}-\bar{g}\right)\right)\right|+\mid w_{\delta}(r) Q_{\bar{g}}\left(\tilde{g}_{\delta}-\bar{g}\right)\right)|+| w_{\delta}(r)\left(R_{\tilde{g}_{\delta}}-R_{\bar{g}}\right) \mid \\
\leq & \left(C_{1}+C_{2}+C_{3}+C_{4}\right) \delta .
\end{aligned}
$$

Here $C_{j}(j=1, \ldots, 4)$ are positive constants independent of $\delta$. Thus we have that $R_{\tilde{g}_{\delta}} \rightarrow R_{\bar{g}}$ in the $C^{0}$-topology on $W$.

Proposition 4.5. (Kobayashi Approximation Theorem [13, Lemma 3.2]) Let $W$ be a compact manifold with boundary $\partial W=M$, and $C \in \mathcal{C}(M)$. Let $\bar{g} \in \mathcal{R i e m}_{C}(W, M)$ be a metric (respectively $\bar{g} \in \mathcal{R i e m}_{C}^{0}(W, M)$ ). Let $g=$ $\left.\bar{g}\right|_{M}$, and let $A_{\bar{g}}$ be the second fundamental form of $M=\partial W$. There exists a family of metrics $\tilde{g}_{\delta} \in \mathcal{R i e m}_{C}(W, M)$ (respectively $\tilde{g}_{\delta} \in \mathcal{R i e m}_{C}^{0}(W, M)$ ) such that

(i) $\tilde{g}_{\delta} \rightarrow \bar{g}$ in the $C^{1}$-topology on $W($ as $\delta \rightarrow 0)$,

(ii) $R_{\tilde{g}_{\delta}} \rightarrow R_{\bar{g}}$ in the $C^{0}$-topology on $W($ as $\delta \rightarrow 0)$, 
(iii) $\tilde{g}_{\delta}$ is conformally equivalent to $\left(g-2 r A_{\bar{g}}\right)+d r^{2}$ on $U_{\varepsilon(\delta)}(M, \bar{g})$,

(iv) $\tilde{g}_{\delta} \equiv \bar{g}$ on $W \backslash U_{\delta}(M, \bar{g})$.

Proof. First, we note that the exponential map exp : $T^{\perp} M \longrightarrow W$ sends $(x, r \cdot \nu) \in T^{\perp} M$ to $\exp _{x}(r \cdot \nu)=(x, r) \in W$. On $M$ we have

$$
\begin{aligned}
& \bar{g}_{00}=\bar{g}\left(\partial_{r}, \partial_{r}\right)=1, \bar{g}_{00}^{\prime}=\partial_{r} \bar{g}\left(\partial_{r}, \partial_{r}\right)=2 \bar{g}\left(\bar{\nabla}_{\partial r} \partial_{r}, \partial_{r}\right)=0, \\
& \bar{g}_{0 i}=\bar{g}\left(\partial_{r}, \partial_{i}\right)=0, \quad \bar{g}_{0 i}^{\prime}=\partial_{r} \bar{g}\left(\partial_{r}, \partial_{i}\right)=\bar{g}\left(\bar{\nabla}_{\partial r} \partial_{r}, \partial_{i}\right)+\bar{g}\left(\partial_{r}, \bar{\nabla}_{\partial r} \partial_{i}\right)=0 \\
& \bar{g}_{i j}=\bar{g}\left(\partial_{i}, \partial_{j}\right)=g_{i j}, \bar{g}_{i j}^{\prime}=\partial_{r} \bar{g}\left(\partial_{i}, \partial_{j}\right)=\bar{g}\left(\bar{\nabla}_{\partial r} \partial_{i}, \partial_{j}\right)+\bar{g}\left(\partial_{i}, \bar{\nabla}_{\partial r} \partial_{j}\right)=-2 A_{i j}
\end{aligned}
$$

Here we used that

$$
\begin{aligned}
& \bar{\nabla}{ }_{\partial r} \partial_{r}=0, \bar{\nabla}_{\partial r} \partial_{i}=-A_{i}^{k} \partial_{k}, \text { which implies } \\
& \bar{g}\left(\partial_{r}, \bar{\nabla}_{\partial r} \partial_{i}\right)=\bar{g}\left(\partial_{r},-A_{i}^{k} \partial_{k}\right)=0, \text { and } \\
& \bar{g}\left(\bar{\nabla}_{\partial r} \partial_{i}, \partial_{j}\right)+\bar{g}\left(\partial_{i}, \bar{\nabla}_{\partial r} \partial_{j}\right) \\
& =\bar{g}\left(\partial_{i},-A_{j}^{k} \partial_{k}\right)+\bar{g}\left(-A_{i}^{k} \partial_{k}, \partial_{j}\right)=-2 A_{i j} .
\end{aligned}
$$

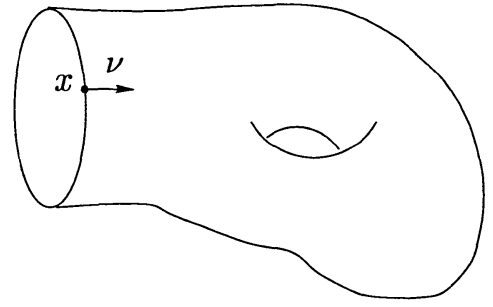

Fig. 4.2.

We define new metrics $\hat{g}$ and $G$ near $M$ as follows:

$$
\begin{aligned}
& \bar{g}(x, r)=\left(g_{i j}(x)-2 r A_{i j}(x)+O\left(r^{2}\right)\right) d x^{i} d x^{j}+O\left(r^{2}\right) d r d x^{i}+d r^{2}, \\
& \hat{g}(x, r):=\left(g_{i j}(x)-2 r A_{i j}(x)\right) d x^{i} d x^{j}+d r^{2}, \\
& G(x, r):=g_{i j}(x) d x^{i} d x^{j}+d r^{2} .
\end{aligned}
$$

Clearly $j_{M}^{1} \bar{g}=j_{M}^{1} \hat{g}$ and, in general, $j_{M}^{1} \bar{g} \neq j_{M}^{1} G$. We notice

$$
\left.R_{\bar{g}}\right|_{M}=R_{G}+2 \operatorname{Ric}_{\bar{g}}(\nu, \nu)+\left|A_{\bar{g}}\right|_{g}^{2}-H_{\bar{g}}^{2}=R_{g}+2 \operatorname{Ric}_{\bar{g}}(\nu, \nu)+\left|A_{\bar{g}}\right|_{g}^{2}-H_{\bar{g}}^{2} .
$$

We define a metric $\check{g}=\left(\check{g}_{i j}\right):=\left(g_{i j}(x)-2 r A_{i j}(x)\right)$ on each hypersuface $M \times\{r\} \subset W$ (for small $r$ ). Then we have

$$
\begin{aligned}
R_{\hat{g}} & =R_{\check{g}}+\frac{3}{4}\left|\partial_{r} \check{g}_{i j}\right|_{\check{g}}^{2}-\check{g}^{i j} \cdot \partial_{r}^{2} \check{g}_{i j}-\frac{1}{4}\left|\check{g}_{i j} \cdot \partial_{r} \check{g}_{i j}\right|^{2} \\
& =R_{g}+3\left|A_{\bar{g}}\right|_{g}^{2}-H_{\bar{g}}^{2}+O(r) \quad \text { near } M, \text { and } \\
R_{\hat{g}} & =R_{g}+3\left|A_{\bar{g}}\right|_{g}^{2}-H_{\bar{g}}^{2} \quad \text { on } M .
\end{aligned}
$$


We choose the conformal metric $\tilde{g}(x, r)=u(x, r)^{\frac{4}{n-2}} \cdot \hat{g}$ so that $j_{M}^{1} \bar{g}=j_{M}^{1} \tilde{g}$ by giving $u$ the boundary conditions:

$$
u(x, 0) \equiv 1, \quad \partial_{r} u(x, 0) \equiv 0 \quad \text { on } M .
$$

We have

$$
-\frac{4(n-1)}{n-2} \Delta_{\hat{g}} u+R_{\hat{g}} u=R_{\tilde{g}} u^{\frac{n+2}{n-2}}, \quad \text { or } \quad \Delta_{\hat{g}} u=-\frac{n-2}{4(n-1)}\left(R_{\tilde{g}} u^{\frac{n+2}{n-2}}-R_{\hat{g}} u\right) .
$$

We specify $\Delta_{\hat{g}} u$ on $M$ :

$$
\begin{aligned}
\Delta_{\hat{g}} u & =\nabla^{\alpha} \partial_{\alpha} u=\hat{g}^{\alpha \beta}\left(\partial_{\alpha} \partial_{\beta} u-\hat{\Gamma}_{\alpha \beta}^{\gamma} \partial_{\gamma} u\right) \\
& =\partial_{r}^{2} u+g^{i j} \partial_{i} \partial_{j} u-\hat{\Gamma}_{00}^{0} \partial_{r} u-\hat{\Gamma}_{00}^{i} \partial_{i} u-g^{i j}\left(\hat{\Gamma}_{i j}^{0} \partial_{r} u+\hat{\Gamma}_{i j}^{k} \partial_{k} u\right)=\partial_{r}^{2} u
\end{aligned}
$$

since $\partial_{i} \partial_{j} u=0, \partial_{r} u=0$ and $\partial_{i} u=0$ on $M$. Here we use the $u(x, 0) \equiv 1$, $\partial_{r} u(x, 0) \equiv 0$ on $M$. Thus we obtain that on $M$

$$
\partial_{r}^{2} u=\Delta_{\hat{g}} u=-\frac{n-2}{4(n-1)}\left(R_{\tilde{g}}-R_{\hat{g}}\right)=-\frac{n-2}{4(n-1)}\left(R_{\tilde{g}}-\left(R_{g}+3\left|A_{\bar{g}}\right|_{g}^{2}-H_{\bar{g}}^{2}\right)\right) .
$$

We let $u(x, r):=1+\frac{1}{2} r^{2} \phi(x)$ near $M$, where

$$
\begin{aligned}
\phi(x) & =-\frac{n-2}{4(n-1)}\left(\left.R_{\bar{g}}\right|_{M}-\left(R_{g}+3\left|A_{\bar{g}}\right|_{g}^{2}-H_{\bar{g}}^{2}\right)\right) \\
& =-\frac{n-2}{4(n-1)}\left(\left.R_{\bar{g}}\right|_{M}-\left.R_{\hat{g}}\right|_{M}\right) .
\end{aligned}
$$

Then the metric

$$
\tilde{g}=u^{\frac{4}{n-2}} \cdot \hat{g}=\left(1+\frac{1}{2} r^{2} \phi(x)\right)^{\frac{4}{n-2}}\left[(g-2 r A)+d r^{2}\right]
$$

is such that $j_{M}^{1} \tilde{g}=j_{M}^{1} \bar{g}$ and $R_{\tilde{g}}=R_{\bar{g}}$ on $M$. We use Proposition 4.4 to define a family of metrics $\tilde{g}_{\delta}$ :

$$
\tilde{g}_{\delta}=\bar{g}+w_{\delta}(r) \cdot(\tilde{g}-\bar{g}) \in \mathcal{R i e m}_{C}(W, M) .
$$

We also notice that $C=\left[\left.\bar{g}\right|_{M}\right]=\left[\left.\hat{g}\right|_{M}\right]=\left[\left.\tilde{g}\right|_{M}\right]=\left[\left.\tilde{g}_{\delta}\right|_{M}\right]$ and

$$
H_{\bar{g}}=0 \Longrightarrow H_{\hat{g}}=0 \Longrightarrow H_{\tilde{g}}=0 \Longrightarrow H_{\tilde{g}_{\delta}}=0
$$

since $A_{\bar{g}}=A_{\hat{g}}, \partial_{r} u=0$ on $M$, and $\tilde{g}=\tilde{g}_{\delta}$ near $M$. Then $\bar{g} \in \mathcal{R i e m}_{C}^{0}(W, M)$ implies that $\tilde{g}_{\delta} \in \mathcal{R i e m}_{C}^{0}(W, M)$.

\subsection{The approximation trick under minimal boundary condi-} tion. One notices that the above results do not allow to use a metric which is conformally equivalent to a product metric near the boundary to approximate the relative Yamabe constant $Y_{\bar{C}}(W, M ; C)$. This is the problem which we address and solve here. 
Theorem 4.6. (Approximation Trick) Let $W$ be a compact manifold with boundary $\partial W=M, C \in \mathcal{C}(M)$. Let $\bar{g} \in \mathcal{R i e m}_{C}^{0}(W, M)$ be a metric. Let $g=\left.\bar{g}\right|_{M}$, and let $A_{\bar{g}}$ be the second fundamental form of $M=\partial W$. Then there exists a family of metrics $\tilde{g}_{\delta} \in \mathcal{R i e m}_{C}^{0}(W, M)$ such that

(i) $\tilde{g}_{\delta} \rightarrow \bar{g}$ in the $C^{0}$-topology on $W($ as $\delta \rightarrow 0)$,

(ii) $R_{\tilde{g}_{\delta}} \rightarrow R_{\bar{g}}$ in the $C^{0}$-topology on $W($ as $\delta \rightarrow 0)$,

(iii) $\tilde{g}_{\delta}$ is conformally equivalent to the metric $g+d r^{2}$ on $U_{\varepsilon(\delta)}(M, \bar{g})$,

(iv) $\tilde{g}_{\delta} \equiv \bar{g}$ on $W \backslash U_{\delta}(M, \bar{g})$.

Remark 6. In order to control the scalar curvature without the minimal boundary condition, one needs the $C^{1}$-convergence of metrics as in Proposition 4.5. Furthermore, when $\bar{g}$ is not totally geodesic on $M$, the metric $\bar{g}$ can never be approximated in the $C^{1}$-topology to a metric which conformally is a product metric near the boundary. However, we emphasize that the convergence in (i) of Theorem 4.6 is the $C^{0}$-convergence only. The minimal boundary condition plays a crucial role to achieve the $C^{0}$-convergence for scalar curvatures in (ii).

Proof. There are two steps in the proof.

Step 1. First, Proposition 4.5 allows us to assume that the metric $\bar{g}$ is such that

$$
\bar{g}=\left(1+\frac{r^{2}}{2} \phi(x)\right)^{\frac{4}{n-2}}\left[\left(g(x)-2 r A_{\bar{g}}(x)\right)+d r^{2}\right]
$$

on a collar $U_{\delta_{0}}(M, \bar{g})$, where $\{x, r\}=\left\{x^{1}, \ldots, x^{n-1}, r\right\}$ denotes a Fermi coordinate system near each point of $M$, and $\phi(x)$ is the smooth function on $M$ defined by (4.1). For each positive $\delta<\delta_{0}$, let $G_{\delta} \in \mathcal{R i e m}_{C}^{0}(W, M)$ be a metric defined by

$$
\begin{aligned}
G_{\delta}(x, r) & =\hat{g}(x, r)+w_{\delta}(r) \cdot(G(x, r)-\hat{g}(x, r)) \\
& =g(x)-2 r\left(1-w_{\delta}(r)\right) \cdot A_{\bar{g}}+d r^{2} .
\end{aligned}
$$

Here $\hat{g}(x, r)$ and $G(x, r)$ are given by

$$
\left.\begin{array}{l}
\hat{g}(x, r)=\left(g(x)-2 r A_{\bar{g}}(x)\right)+d r^{2}, \\
G(x, r)=g(x)+d r^{2}
\end{array}\right\} \text { on } U_{\delta}(M, \bar{g}) .
$$


We also let $\check{g}_{\delta}(x, r)=g(x)-2 r\left(1-w_{\delta}(r)\right) \cdot A_{\bar{g}}(x)$ on $U_{\delta}(M, \bar{g})$. It then follows from Lemma 4.2 that near $M$ the scalar curvature of the metric $G_{\delta}$ satisfies

$$
\begin{aligned}
R_{G_{\delta}}= & R_{\check{g}}+\frac{3}{4}\left|\partial_{r}\left(\check{g}_{\delta}\right)_{i j}\right|_{\check{g}_{\delta}}^{2}-\check{g}_{\delta}^{i j} \cdot \partial_{r}^{2}\left(\check{g}_{\delta}\right)_{i j}-\frac{1}{4}\left|\check{g}_{\delta}^{i j} \cdot \partial_{r}\left(\check{g}_{\delta}\right)_{i j}\right|^{2} \\
= & R_{g}+3\left(1-w_{\delta}(t)\right)^{2}\left|A_{\bar{g}}\right|_{g}^{2}-\left(1-w_{\delta}(t)\right)^{2} H_{\bar{g}}^{2} \\
& -\left(4 \dot{w}_{\delta}(r)+2 r \cdot \ddot{w}_{\delta}(r)\right) H_{\bar{g}}+O(\delta) .
\end{aligned}
$$

We use the minimal boundary condition $H_{\bar{g}}=0$ to obtain

$$
R_{G_{\delta}}=R_{g}+3\left(1-w_{\delta}(r)\right)^{2}\left|A_{\bar{g}}\right|_{g}^{2}+O(\delta) \quad \text { near } M .
$$

Step 2. We now define the metric $\tilde{g}_{\delta} \in \operatorname{Riem}_{C}^{0}(W, M)$ as follows:

$$
\tilde{g}_{\delta}(x, r)=\left(1+\frac{r^{2}}{2} \phi_{\delta}(x, r)\right)^{\frac{4}{n-2}} \cdot G_{\delta}(x, r)
$$

on $U_{\delta}(M, \bar{g})$ with

$$
\phi_{\delta}(x, r)=\phi(x)-\frac{3(n-2)}{4(n-1)}\left(2-w_{\delta}(r)\right) w_{\delta}(r)\left|A_{\bar{g}}\right|_{g}^{2} .
$$

We obtain that the assertions (iii) and (iv) hold since $G_{\delta}=g+d r^{2}$ on the collar $U_{\varepsilon(\delta)}(M, \bar{g})$, and $G_{\delta}=\hat{g}$, and $\phi_{\delta}=\phi$ outside of the collar $U_{\delta}(M, \bar{g})$. By construction

$$
G_{\delta} \longrightarrow \hat{g}, \quad \text { and } \quad \frac{r^{2}}{2} \phi_{\delta}(x, r) \longrightarrow \frac{r^{2}}{2} \phi(x)
$$

in the $C^{0}$-topology on $W$ as $\delta \longrightarrow 0$. Thus the assertion (i) holds.

Finally, the scalar curvature $R_{\tilde{g}_{\delta}}$ is given by

$$
\begin{aligned}
& R_{\tilde{g}_{\delta}}=\left(1+\frac{r^{2}}{2} \phi_{\delta}(x, r)\right)^{-\frac{n+2}{n-2}}\left[-\frac{4(n-2)}{n-1} \Delta_{G_{\delta}}\left(1+\frac{r^{2}}{2} \phi_{\delta}(x, r)\right)\right. \\
& \left.+R_{G_{\delta}}\left(1+\frac{r^{2}}{2} \phi_{\delta}(x, r)\right)\right] \\
& =\left(1+O\left(\delta^{2}\right)\right)\left[-\frac{4(n-2)}{n-1} \phi+3\left(2-w_{\delta}(r)\right) \cdot w_{\delta}(r) \cdot\left|A_{\bar{g}}\right|_{g}^{2}+R_{G_{\delta}}+O(\delta)\right] \\
& =R_{G_{\delta}}+\left(R_{\bar{g}}-R_{g}-3\left|A_{\bar{g}}\right|_{g}^{2}\right)+3\left(2-w_{\delta}(r)\right) \cdot w_{\delta}(r) \cdot\left|A_{\bar{g}}\right|_{g}^{2}+O(\delta) \\
& =R_{g}+3\left(1-w_{\delta}(r)\right)^{2}\left|A_{\bar{g}}\right|_{g}^{2}+\left(R_{\bar{g}}-R_{g}-3\left|A_{\bar{g}}\right|_{g}^{2}\right) \\
& +3\left(2-w_{\delta}(r)\right) w_{\delta}(r)\left|A_{\bar{g}}\right|_{g}^{2}+O(\delta) \\
& =R_{\bar{g}}+O(\delta) \quad \text { on } W \text { as } \delta \longrightarrow 0 \text {. }
\end{aligned}
$$


This implies the assertion (ii) and completes the proof of Theorem 4.6.

\section{Gluing Theorems.}

5.1. Setting. Here we would like to analyze the gluing procedure for manifolds equipped with conformal structures. Let $W_{1}, W_{2}$ be two compact manifolds of $\operatorname{dim} W_{1}=\operatorname{dim} W_{2} \geq 3$ with boundaries

$$
\partial W_{1}=M_{1}=M_{0} \sqcup M, \quad \text { and } \quad \partial W_{2}=M_{2}=M_{0} \sqcup M^{\prime}
$$

endowed with conformal classes $C_{1}=C_{0} \sqcup C \in \mathcal{C}\left(M_{1}\right), C_{2}=C_{0} \sqcup C^{\prime} \in \mathcal{C}\left(M_{2}\right)$, where $C_{0} \in \mathcal{C}\left(M_{0}\right), C \in \mathcal{C}(M), C^{\prime} \in \mathcal{C}\left(M^{\prime}\right)$. Let $W=W_{1} \cup_{M_{0}}\left(-W_{2}\right)$ be the union of of $W_{1}$ and $W_{2}$ along $M_{0}$ (see Fig. 5.1).

Remark 7. The boundary of the manifold $W$ is $\partial W=M \sqcup M^{\prime}$ with appropriate orientation. We consider both cases when $\partial W=\emptyset$ and $\partial W \neq \emptyset$.

Recall that a conformal class $C \in \mathcal{C}(M)$ is positive if $Y_{C}(M)>0$.

Theorem 5.1. Let $C_{0} \in \mathcal{C}\left(M_{0}\right)$ be a positive conformal class, and $Y\left(W_{j}, M_{j} ; C_{j}\right)>0$ for $j=1,2$. Then $Y\left(W, \partial W ; C \sqcup C^{\prime}\right)>0$.

Remark 8. We do not assume that the conformal classes $C \in \mathcal{C}(M), C^{\prime} \in$ $\mathcal{C}\left(M^{\prime}\right)$ are positive.

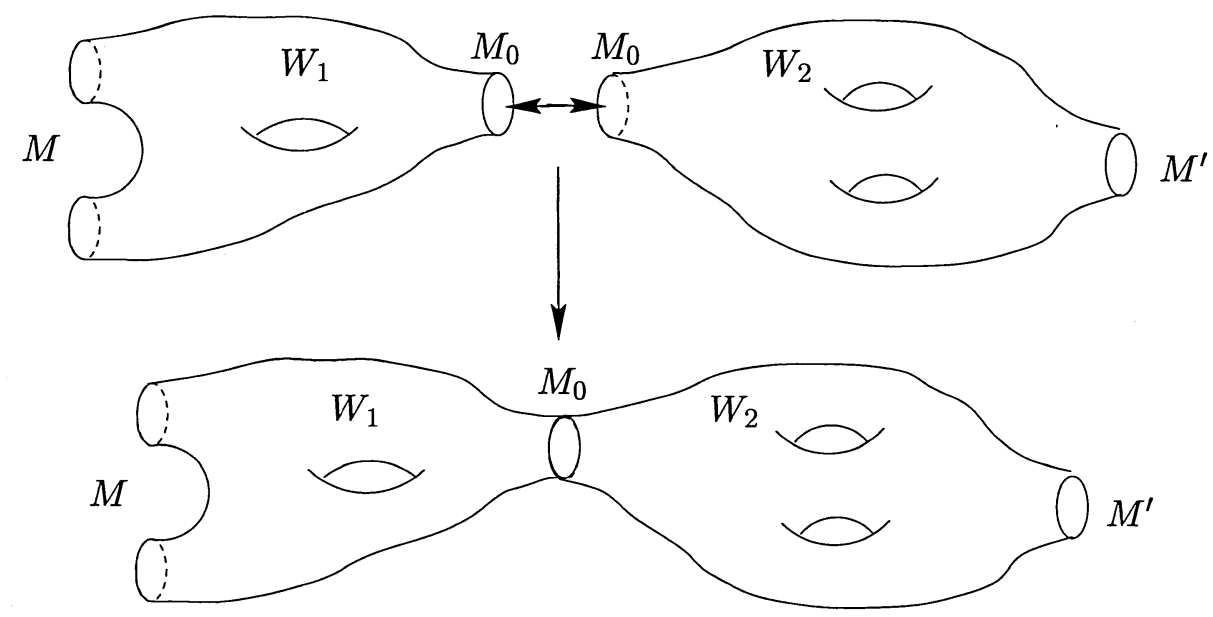

Fig. 5.1. Manifold $W=W_{1} \cup_{M_{0}}\left(-W_{2}\right)$.

Proof of Theorem 5.1. There are four steps in the proof. 
Step 1. First we notice that since $C_{0} \in \mathcal{C}\left(M_{0}\right)$ is a positive conformal class, there exists a metric $h \in C_{0}$ on $M_{0}$ with $R_{h}>0$. The metric $h$ do not have to be a Yamabe metric. We fix the metric $h$. The condition $Y\left(W_{j}, M_{j} ; C_{j}\right)>0$ (for $\left.j=1,2\right)$ implies that there exist conformal classes $\bar{C}_{j}$ on $W_{j}$ such that $\partial \bar{C}_{j}=C_{j}$, i.e. $\left(\bar{C}_{j}, C_{j}\right) \in \mathcal{C}\left(W_{j}, M_{j}\right)$. We denote

$$
Y_{\bar{C}_{j}}=Y_{\bar{C}_{j}}\left(W_{j}, M_{j} ; C_{j}\right)>0, \quad j=1,2 .
$$

Choose metrics $\bar{g}_{j} \in \bar{C}_{j}$ with $\left.\bar{g}_{j}\right|_{M_{0}}=h$. Moreover, we may assume that $\bar{g}_{j} \in \bar{C}_{j}^{0}$ (i.e. $H_{\bar{g}_{j}} \equiv 0$ on $M_{j}$ ).

Remark 9. (1) The metrics $\bar{g}_{j} \in \bar{C}_{j}^{0}$ do not have to be relative Yamabe metrics. Moreover, their scalar curvature $R_{\bar{g}_{j}}$ is not positive, in general.

(2) The union $\bar{C}_{1}^{0} \cup_{C} \bar{C}_{2}^{0}$ does not make sense as a conformal class on $W$ since this union, in general, fails to be smooth along $M_{0}$.

Step 2. Theorem 4.6 and (4.2), (4.3) imply that for any $\varepsilon>0$ there exist conformal classes $\hat{C}_{j}$ on $W_{j}$ and metrics $\hat{g}_{j} \in \hat{C}_{j}(j=1,2)$ such that

$$
\left\{\begin{array}{l}
\partial \hat{C}_{j}=C_{j}, \\
\hat{g}_{j} \sim \bar{g}_{j}, \\
R_{\hat{g}_{j}} \sim R_{\bar{g}_{j}}, \\
\hat{g}_{j}=\left(1+\frac{r^{2}}{2} f_{j}\right)^{\frac{4}{n-2}} \cdot\left(h+d r^{2}\right) \text { near } M_{j} \text { in } W_{j} .
\end{array}\right.
$$

Here the function $f_{j}$ is defined by

$$
f_{j}=-\frac{n-2}{4(n-1)}\left(\left.R_{\bar{g}_{j}}\right|_{M_{0}}-R_{h}\right) \quad \text { on } M_{0} \text { in each } W_{j} .
$$

From now on we only need the conditions $Y_{\hat{C}_{j}}>0(j=1,2)$. Therefore we may assume that $f_{j}<0$ on $M_{0}$ since the relative Yamabe constant $Y_{\hat{C}_{j}}$ is invariant under pointwise conformal change.

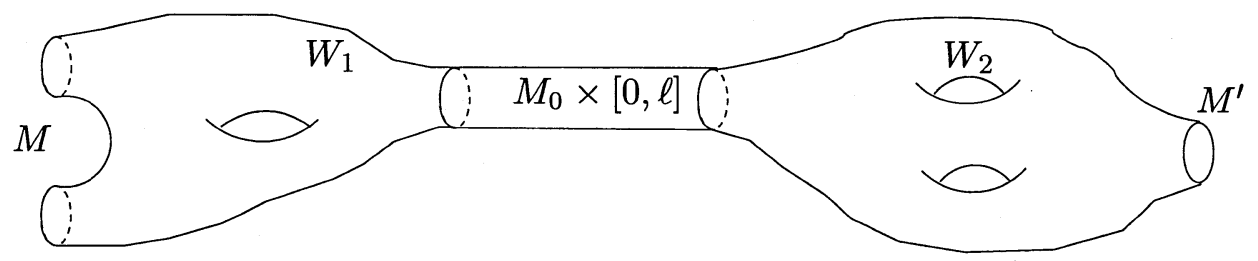

Fig. 5.2. Manifold $X=W_{1} \cup_{M_{0}}\left(M_{0} \times[0, \ell]\right) \cup_{M_{0}}\left(-W_{2}\right)$. 
Let $\ell$ be a positive constant. We define the manifold $X$ which is diffeomorphic to $W$ as follows (see Fig. 5.2):

$$
X=W_{1} \cup_{M_{0}}\left(M_{0} \times[0, \ell]\right) \cup_{M_{0}}\left(-W_{2}\right)
$$

Now we need the cut-off function $w_{\delta}$ defined in Lemma 4.2. Then for each $\delta, 0<\delta<\frac{\ell}{2}$, we define a metric $\tilde{g}$ on $X$ as follows.

$$
\tilde{g}= \begin{cases}\hat{g}_{1} & \text { on } W_{1}, \\ \hat{g}_{2} & \text { on } W_{2}, \\ \left(1+\frac{r^{2}}{2} w_{\delta}(r) f_{1}\right)^{\frac{4}{n-2}}\left(h+d r^{2}\right) & \text { on } M_{0} \times[0, \delta], \\ h+d r^{2} & \text { on } M_{0} \times[\delta, \ell-\delta], \\ \left(1+\frac{(\ell-r)^{2}}{2} w_{\delta}(\ell-r) f_{2}\right)^{\frac{4}{n-2}}\left(h+d r^{2}\right) & \text { on } M_{0} \times[\ell-\delta, \ell] .\end{cases}
$$

Clearly $\tilde{g}$ is a smooth metric on $X \cong W$. Let $\tilde{C}=[\tilde{g}] \in \mathcal{C}(W)$.

Remark 10. The metric $\tilde{g}$ does not have positive scalar curvature.

Step 3. Let $j=1,2$. Denote by $\nu_{j}$ the first eigenvalue of the Yamabe operator on $W_{j}$ for the Neumann boundary condition. Then

$$
\nu_{j}=\inf _{\substack{u \in C^{\infty}\left(W_{j}\right) \\ u \neq 0}} \frac{\int_{W_{j}}\left(\frac{4(n-1)}{n-2}|d u|_{\hat{g}_{j}}^{2}+R_{\hat{g}_{j}} u^{2}\right) d \sigma_{\hat{g}_{j}}}{\int_{W_{j}} u^{2} d \sigma_{\hat{g}_{j}}} .
$$

The relative Yamabe constants $Y_{\hat{C}_{j}}>0$ since $Y_{\hat{C}_{j}}>Y_{\bar{C}_{j}}-\varepsilon, j=1,2$. Thus it follows that $\nu_{j}>0$. Notice that the conditions $R_{h}>0$ on $M$ and $f_{j}<0$ $(j=1,2)$ imply that $R_{\tilde{g}}>0$ on the cylinder $M_{0} \times[0, \ell]$ for small $\delta>0$.

Let $\nu_{c y l}$ be the first eigenvalue of the Yamabe operator on $M_{0} \times[0, \ell]$ for the Neumann boundary condition. We have

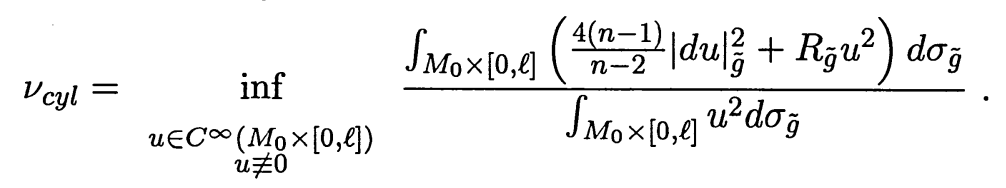

It follows that $\nu_{c y l}>0$ since $R_{\tilde{g}}>0$.

Step 4. Let $\nu$ be the first eigenvalue of the Yamabe operator on $X \cong W$ for the Neumann boundary condition, which is equal to

$$
\nu=\inf _{\substack{u \in C^{\infty}(X) \\ u \neq 0}} \frac{\int_{X}\left(\frac{4(n-1)}{n-2}|d u|_{\tilde{g}}^{2}+R_{\tilde{g}} u^{2}\right) d \sigma_{\tilde{g}}}{\int_{X} u^{2} d \sigma_{\tilde{g}}} .
$$


We conclude that $\nu \geq \min \left\{\nu_{1}, \nu_{2}, \nu_{c y l}\right\}>0$ by [5, pp. 18-19]. The condition $\nu>0$ is equivalent that there exists a metric $\check{g} \in[\tilde{g}]$ such that $R_{\check{g}}>0$ on $X \cong W$ and $H_{\check{g}}=0$ on $\partial X=\partial W$. Thus $Y_{[\check{g}]}\left(X, \partial X ; C \sqcup C^{\prime}\right)>0$, and this implies $Y\left(W, M \sqcup M^{\prime} ; C \sqcup C^{\prime}\right)>0$.

Remark 11. Notice that $\ell>0$ could be chosen to be small under appropriate choice of $\delta>0$.

5.3. Manifolds with positive relative Yamabe invariant. Here we show that there are many examples of manifolds with positive relative Yamabe invariant. We start with a closed manifold $N$ of $\operatorname{dim} N \geq 3$ with $Y(N)>0$. We choose an embedded small disk $D^{n} \subset N$ centered at $x_{0} \in N$, then $\partial\left(N \backslash \operatorname{int}\left(D^{n}\right)\right)=S^{n-1}$. Let $C_{\text {can }} \in \mathcal{C}\left(S^{n-1}\right)$ be the standard conformal class.

Theorem 5.2. Let $N$ be a closed manifold of $\operatorname{dim} N \geq 3$ with $Y(N)>0$. Then $Y\left(N \backslash \operatorname{int}\left(D^{n}\right), S^{n-1} ; C_{\text {can }}\right)>0$.

Proof. We use [13, Corollary 3.5.] to choose a conformal class $\bar{C} \in \mathcal{C}(N)$ with the Yamabe constant $Y_{\bar{C}}(N)>0$ and a metric $\bar{g} \in \bar{C}$ such that

- $\bar{g}$ is conformally flat near $x_{0} \in N$,

- $R_{\bar{g}}>0$ on $N$.

Thus (as it was observed by Gromov-Lawson [9]), there exists a metric $\hat{g}$ on the manifold $N \backslash \operatorname{int}\left(D^{n}\right)$ such that

- $\partial[\hat{g}]=C_{\text {can }} \in \mathcal{C}\left(S^{n-1}\right)$,

- $R_{\hat{g}}>0$ on $N \backslash \operatorname{int}\left(D^{n}\right)$,

- $\hat{g}=g_{S^{n-1}}+d r^{2}$ near $S^{n-1}=\partial\left(N \backslash \operatorname{int}\left(D^{n}\right)\right),\left[g_{S^{n-1}}\right]=C_{\text {can }}$.

Thus $Y_{[\hat{g}]}\left(N \backslash \operatorname{int}\left(D^{n}\right), S^{n-1} ; C_{\text {can }}\right)>0$ and $Y\left(N \backslash \operatorname{int}\left(D^{n}\right), S^{n-1} ; C_{\text {can }}\right)>0$.

5.4. The double. Let $W$ be a compact manifold with $\partial W=M=$ $M_{0} \sqcup M_{1}$. We consider the manifold $X=W \cup_{M_{0}}(-W)$ which is the double of $W$ along $M_{0}$ (see Fig. 5.3).

Remark 12. The other boundary component $M_{1}$ of $\partial W$ may be empty or not. 


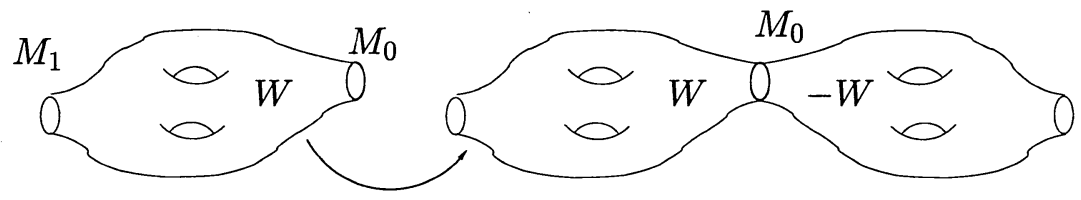

Fig. 5.3.

Theorem 5.3. Let $W, X$ be manifolds as above, and $C=C_{0} \sqcup C_{1} \in \mathcal{C}(\partial W)$, where $C_{0} \in \mathcal{C}\left(M_{0}\right), C_{1} \in \mathcal{C}\left(M_{1}\right)$. Then

(1) $Y\left(X, M_{1} \sqcup\left(-M_{1}\right) ; C_{1} \sqcup C_{1}\right) \geq \begin{cases}Y(W, M ; C) & \text { if } Y(W, M ; C)>0, \\ 2^{\frac{2}{n}} Y(W, M ; C) & \text { if } Y(W, M ; C) \leq 0 .\end{cases}$

$$
Y\left(X, M_{1} \sqcup\left(-M_{1}\right)\right) \geq \begin{cases}Y(W, M) & \text { if } Y(W, M)>0, \\ 2^{\frac{2}{n}} Y(W, M) & \text { if } Y(W, M) \leq 0 .\end{cases}
$$

Proof. Let $\bar{C}$ be a conformal class on $W$ with $\partial \bar{C}=C$. It is enough to prove the following inequality:

(5.1) $Y\left(X, M_{1} \sqcup\left(-M_{1}\right) ; C_{1} \sqcup C_{1}\right) \geq \begin{cases}Y_{\bar{C}}(W, M ; C) & \text { if } Y_{\bar{C}}(W, M ; C)>0, \\ 2^{\frac{2}{n}} Y_{\bar{C}}(W, M ; C) & \text { if } Y_{\bar{C}}(W, M ; C) \leq 0 .\end{cases}$

We choose a metric $\bar{g} \in \bar{C}^{0}$, and let $g=\left.\bar{g}\right|_{M}$. We notice that, for a generic conformal class $\bar{C}$, any metric $\bar{g} \in \bar{C}^{0}$ could not be extended smoothly to $X$. It then follows from Theorem 4.6 that, for any small $\varepsilon>0$, there exists a metric $\hat{g} \in \hat{C}$ such that $\left.\hat{g}\right|_{M}=g=\left.\bar{g}\right|_{M}$ and

$$
\left\{\begin{array}{l}
\hat{g} \sim \bar{g} C^{0} \text {-close on } W \\
\left|R_{\hat{g}}-R_{\bar{g}}\right|<\varepsilon \text { on } W \\
\hat{g}(x, r)=\left(1+\frac{r^{2}}{2} f(x)\right)^{\frac{4}{n-2}}\left(g(x)+d r^{2}\right) \quad \text { near } M \subset W .
\end{array}\right.
$$

Thus we obtain that

$$
\begin{gathered}
\left|Y_{\hat{C}}(W, M ; C)-Y_{\bar{C}}(W, M ; C)\right| \leq K \varepsilon, \\
Y_{\hat{C}}(W, M ; C)>0 \text { if } Y_{\bar{C}}(W, M ; C)>0
\end{gathered}
$$

where $K>0$ is a constant independent of $\varepsilon$. We define $\tilde{g}:=\hat{g} \cup \hat{g}$ on $X=W \cup_{M_{0}}(-W)$. The metric $\tilde{g}$ is smooth by construction. Let $\tilde{C}:=$ 
$\hat{C} \cup \hat{C} \in \mathcal{C}(X)$. Now for any $u \in C^{\infty}(X), u \not \equiv 0$, we define $Q_{(X, \tilde{g})}(u)$ as

$$
\begin{aligned}
& Q_{(X, \tilde{g})}(u)=\frac{E_{(X, \tilde{g})}(u)}{\left(\int_{X}|u|^{\frac{2 n}{n-2}} d \sigma_{\tilde{g}}\right)^{\frac{n-2}{n}}}, \text { where } \\
& E_{(X, \tilde{g})}(u)=\int_{X}\left(\frac{4(n-1)}{n-2}|d u|^{2}+R_{\tilde{g}} u^{2}\right) d \sigma_{\tilde{g}} .
\end{aligned}
$$

We denote $\alpha=\int_{W}|u|^{\frac{2 n}{n-2}} d \sigma_{\hat{g}}, \beta=\int_{-W}|u|^{\frac{2 n}{n-2}} d \sigma_{\hat{g}}$. It is enough to consider the case $\alpha, \beta>0$ and $\alpha+\beta=1$. Then

$$
\begin{aligned}
Q_{(X, \tilde{g})}(u) & =\frac{E_{(W, \hat{g})}(u)+E_{(-W, \hat{g})}(u)}{(\alpha+\beta)^{\frac{n-2}{n}}} \\
& =\alpha^{\frac{n-2}{n}} \cdot \frac{E_{(W, \hat{g})}(u)}{\alpha^{\frac{n-2}{n}}}+\beta^{\frac{n-2}{n}} \cdot \frac{E_{(-W, \hat{g})}(u)}{\beta^{\frac{n-2}{n}}} \\
& \geq\left(\alpha^{\frac{n-2}{n}}+(1-\alpha)^{\frac{n-2}{n}}\right) Y_{\hat{C}}(W, M ; C)
\end{aligned}
$$

for any $\alpha \in(0,1)$. Let $Y:=Y_{\tilde{C}}\left(X, M_{1} \sqcup\left(-M_{1}\right) ; C_{1} \sqcup C_{1}\right)$. From (5.2), this implies that

$$
\begin{aligned}
Y & \geq \inf _{0 \leq \alpha \leq 1}\left(\alpha^{\frac{n-2}{n}}+(1-\alpha)^{\frac{n-2}{n}}\right) Y_{\hat{C}}(W, M ; C) \\
& \geq \begin{cases}Y_{\hat{C}}(W, M ; C) & \text { if } Y_{\hat{C}}(W, M ; C)>0, \\
2^{\frac{2}{n}} Y_{\hat{C}}(W, M ; C) & \text { if } Y_{\hat{C}}(W, M ; C) \leq 0,\end{cases} \\
& \geq \begin{cases}Y_{\bar{C}}(W, M ; C)-K \varepsilon & \text { if } Y_{\bar{C}}(W, M ; C)>0, \\
2^{\frac{2}{n}}\left(Y_{\bar{C}}(W, M ; C)-K \varepsilon\right) & \text { if } Y_{\bar{C}}(W, M ; C) \leq 0 .\end{cases}
\end{aligned}
$$

We let $\varepsilon \rightarrow 0$ to obtain (5.1).

In the case of double manifolds, we obtain the following generalization of Kobayashi's inequality [13, Theorem 2].

Corollary 5.4. Let $\partial W=M$, and let $X=W \cup_{M}(-W)$ be the double of $W, \partial X=\emptyset$. Then

$$
Y(X) \geq \begin{cases}Y(W, M) & \text { if } Y(W, M)>0 \\ 2^{\frac{2}{n}} Y(W, M) & \text { if } Y(W, M) \leq 0 .\end{cases}
$$

In particular, if $Y(X) \leq 0$, then $Y(W, M) \leq 2^{-\frac{2}{n}} Y(X) \leq 0$. 


\section{Non-positive and negative relative Yamabe invariant.}

6.1. Enlargeable manifolds. Let $N$ be a closed manifold of $\operatorname{dim} N=n$ and $D^{n} \subset N$ an embedded disk. We define the manifold $W=N \backslash \operatorname{int}\left(D^{n}\right)$ with $\partial W=S^{n-1}$.

Remark 13. Let $N$ be an enlargeable closed manifold (see [10]). Then the manifold $N \#(-N)=W \cup_{S^{n-1}}(-W)$ is also enlargeable. Thus $Y(N \#(-N)) \leq 0$.

Corollary 6.1. Let $N$ be an enlargeable closed manifold. Then

$$
2^{\frac{2}{n}} Y\left(N \backslash \operatorname{int}\left(D^{n}\right), S^{n-1}\right) \leq Y(N \#(-N)) .
$$

In particular, $Y\left(N \backslash \operatorname{int}\left(D^{n}\right), S^{n-1}\right) \leq 0$.

Example. Let $T^{n}$ be a torus, and let $\mathbf{H}^{n}$ be a hyperbolic space and $\Gamma$ a discrete group acting freely on $\mathbf{H}^{n}$ such that $\mathbf{H}^{n} / \Gamma$ is a compact manifold. Then we have $Y\left(T^{n} \backslash \operatorname{int}\left(D^{n}\right), S^{n-1}\right) \leq 0, Y\left(\left(\mathbf{H}^{n} / \Gamma\right) \backslash \operatorname{int}\left(D^{n}\right), S^{n-1}\right) \leq 0$.

Remark 14. Let $W_{j}$ be a compact smooth $n$-manifold with boundary $\partial W_{j}=M_{j}$, for $j=1,2$. Let $(W, M)=\left(W_{1}, M_{1}\right) \sqcup\left(W_{2}, M_{2}\right)$ be the disjoint union of $W_{1}$ and $W_{2}$. Let $C=C_{1} \sqcup C_{2}$ be a conformal class on $M_{1} \sqcup M_{2}$. Similarly to the case of closed manifolds, we can show that the same equality as that of [13, Corollary 1.11] holds for the relative Yamabe invariants $Y(W, M ; C)$ and $Y\left(W_{j}, M_{j} ; C_{j}\right)(j=1,2)$.

6.2. Four-manifolds with negative relative Yamabe invariant. Now we would like to construct a family of 4-manifolds with strictly negative relative Yamabe invariant. Let $X$ be a minimal closed symplectic 4-manifold of general type (see [16]) with an involution $\iota$. Assume that the fixed point set $\operatorname{Fix}(\iota)$ of $\iota$ is an oriented closed 3-manifold $M$ (possibly disconnected). First, we notice the following fact.

Lemma 6.2. The complement $X \backslash M$ consists of two connected components. Moreover, the manifold $X=W \cup_{M}(-W)$ is the double of the closure $W$ of a connected component of $X \backslash M$.

Proof. Since $X$ is oriented and $M$ is an oriented submanifold of codimension one, the normal bundle $\nu(M)$ of $M$ in $X$ is trivial, and the differential $d \iota$ of the involution $\iota$ satisfies $\left.d \iota\right|_{T M}=I d,\left.\quad d \iota\right|_{\nu(M)}=-I d$. In particular, $\iota$ is 
orientation-reversing. Now we choose a metric $\bar{g}$ such that $\iota$ is an isometry of $(X, \bar{g})$. Then, it follows from [14, Theorem 5.1] that $M$ is totally geodesic. Let $\hat{X}$ denote the canonical compactification of the complement $X \backslash M$ with the boundary $\partial \hat{X}=M \sqcup(-M)$ (see Fig. 6.1). Notice that the metric $\bar{g}$ can be extended to $\hat{X}$ naturally.
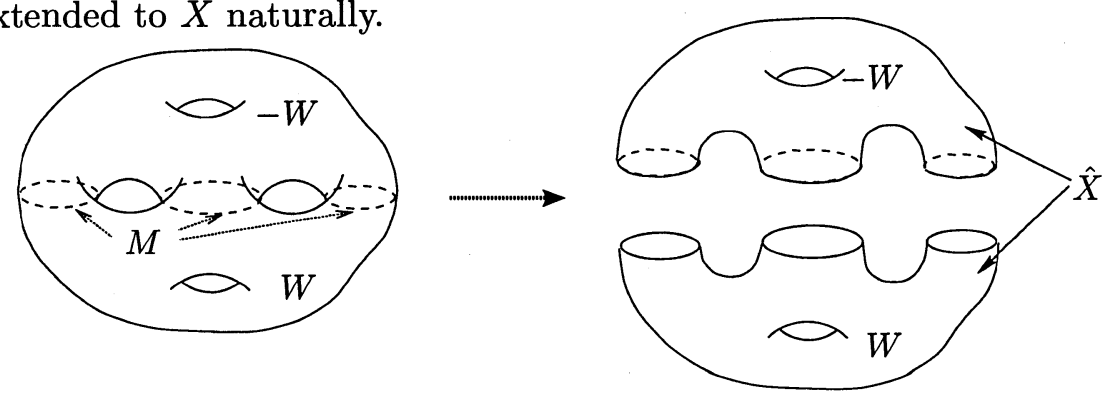

Fig. 6.1.

Suppose that $X \backslash M$ is connected. Let $M_{1}$ be a connected component of $M$. Then there exists a shortest geodesic $c:[0,1] \rightarrow \hat{X}$ joining $M_{1}$ and $-M_{1}$. Note that $c((0,1)) \subset X \backslash M$, and the tangent vectors $c^{\prime}(0)$ and $c^{\prime}(1)$ are perpendicular to $M_{1}$ and $-M_{1}$, respectively. Since $\iota$ is an involutive isometry, the curve $\iota \circ c:[0,1] \rightarrow \hat{X}$ is also a geodesic from $c(1) \in\left(-M_{1}\right)$ to $c(0) \in M_{1}$ such that the tangent vectors $(\iota \circ c)^{\prime}(0)=-c^{\prime}(1)$ and $(\iota \circ c)^{\prime}(1)=-c^{\prime}(0)$ are respectively perpendicular to $-M_{1}$ and $M_{1}$. Hence, $(\iota \circ c)([0,1])=c([0,1])$, and then $\iota\left(c\left(\frac{1}{2}\right)\right)=c\left(\frac{1}{2}\right) \in X \backslash M$. This contradicts to the fact that $\operatorname{Fix}(\iota)=M$. Therefore, $X \backslash M$ is disconnected. Let $W$ be the closure of a connected component $X_{1}$ of $X \backslash M$ satisfying $\partial W=M$. Then $\iota(W)$ is also the closure of a connected component $\iota\left(X_{1}\right)$ of $X \backslash M$. The conditions $\left.d \iota\right|_{T M}=I d,\left.d \iota\right|_{\nu(M)}=-I d$ imply that $X_{1} \cap \iota\left(X_{1}\right)=\emptyset$. Then we have that $X=W \cup_{M}(-\iota(W))=W \cup_{M}(-W)$, and $X \backslash M$ consists of two connected components $X_{1}$ and $-\iota\left(X_{1}\right)$.

Let $W \# \ell\left(S^{1} \times S^{3}\right)$ be the connected sum of $W$ with $\ell$ copies of $S^{1} \times S^{3}$ (where $\ell \geq 0$ ). We combine the estimates given in [16], [21] (cf. [27]) of the Yamabe invariant in dimension four with Corollary 5.4 to obtain the following result.

Theorem 6.3. Let $X$ and $W$ be the 4-manifolds as above. Then the relative Yamabe invariant $Y\left(W \# \ell\left(S^{1} \times S^{3}\right), M\right)$ is strictly negative. Moreover,

$$
\begin{aligned}
Y\left(W \# \ell\left(S^{1} \times S^{3}\right), M\right) & \leq \frac{1}{\sqrt{2}} Y\left(X \# 2 \ell\left(S^{1} \times S^{3}\right)\right)=\frac{1}{\sqrt{2}} Y(X) \\
& \leq-4 \pi \sqrt{2 \chi(X)+3 \sigma(X)}<0
\end{aligned}
$$


where $\chi(X)$ and $\sigma(X)$ are respectively the Euler characteristic and the signature of $X$.

Proof. Since there is an orientation-reversing diffeomorphism of $S^{1} \times S^{3}$, then there is a diffeomorphism

$$
X \# 2 \ell\left(S^{1} \times S^{3}\right) \cong X \# \ell\left(S^{1} \times S^{3}\right) \# \ell\left(-\left(S^{1} \times S^{3}\right)\right) .
$$

From [16, Theorem 4], [21, Proposition 3], Corollary 5.4 and Lemma 6.2, one has

$$
\begin{aligned}
Y\left(W \# \ell\left(S^{1} \times S^{3}\right), M\right) & \leq \frac{1}{\sqrt{2}} Y\left(X \# \ell\left(S^{1} \times S^{3}\right) \# \ell\left(-\left(S^{1} \times S^{3}\right)\right)\right) \\
& =\frac{1}{\sqrt{2}} Y\left(X \# 2 \ell\left(S^{1} \times S^{3}\right)\right)=\frac{1}{\sqrt{2}} Y(X) \\
& \leq-4 \pi \sqrt{2 \chi(X)+3 \sigma(X)}<0 .
\end{aligned}
$$

This completes the proof of Theorem 6.3.

Let $N$ be a product $\Sigma_{k_{1}} \times \Sigma_{k_{2}}$ of closed Riemann surfaces of genus $k_{1}, k_{2} \geq$ 2 with a product metric $\bar{g}=g_{1} \times g_{2}$ of $g_{1}$ on $\Sigma_{k_{1}}$ and $g_{2}$ on $\Sigma_{k_{2}}$, respectively, of constant negative curvature -1 . Assume that the hyperbolic metric $g_{1}$ admits an involutive isometry $\phi$ of $\left(\Sigma_{k_{1}}, g_{k_{1}}\right)$ such that $\operatorname{Fix}(\phi)$ consists of $k_{1}+1$ disjoint simple closed geodesics (see Fig. 6.2). We remark that such hyperbolic metric always exists on $\Sigma_{k_{1}}$ provided $k_{1} \geq 2$.
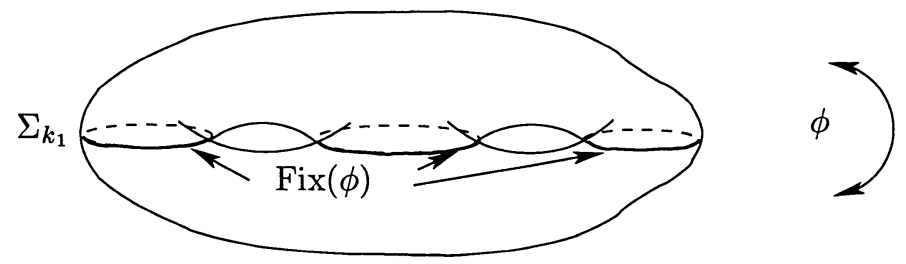

Fig. 6.2.

Then the map $\iota:=\phi \times I d: N=\Sigma_{k_{1}} \times \Sigma_{k_{2}} \rightarrow N$ is also an involutive isometry with $\operatorname{Fix}(\iota)=\operatorname{Fix}(\phi) \times \Sigma_{k_{2}}$ consisting of $k_{1}+1$ disjoint closed totally geodesic 3 -submanifolds. Let $W$ denote the closure of a connected components of $N \backslash \operatorname{Fix}(\iota)$ satisfying $N=W \cup_{\partial W}(-W)$. We rewrite

$$
W \# \ell\left(S^{1} \times S^{3}\right)=\left(W \backslash \bigcup_{j=1}^{\ell} \operatorname{int}\left(D_{j}^{4}\right)\right) \cup_{\partial} \ell\left(\left(S^{1} \times S^{3}\right) \backslash \operatorname{int}\left(D^{4}\right)\right)
$$


where $D_{j}^{4}, j=1, \ldots, \ell$, are disjoint small 4-disks inside $\operatorname{int}(W)$, and $\partial=$ $\cup_{j=1}^{\ell} \partial D_{j}^{4}$. Then there is an involution $\hat{\imath}$ of the manifold

$$
N \# 2 \ell\left(S^{1} \times S^{3}\right)=\left(W \# \ell\left(S^{1} \times S^{3}\right)\right) \cup_{\partial W}\left(-\left(W \# \ell\left(S^{1} \times S^{3}\right)\right)\right)
$$

with $\operatorname{Fix}(\hat{\iota})=\operatorname{Fix}(\iota)=\partial W$, and $\hat{\iota}=\iota$ on

$$
\left(W \backslash \bigcup_{j=1}^{\ell} \operatorname{int}\left(D_{j}^{4}\right)\right) \cup_{\partial W}\left(-\left(W \backslash \bigcup_{j=1}^{\ell} \operatorname{int}\left(D_{j}^{4}\right)\right)\right) .
$$

With these understood, we obtain the following result.

Corollary 6.4. For any $\ell \geq 0$ the relative Yamabe invariant of the manifold $W \# \ell\left(S^{1} \times S^{3}\right)$ is given by

$$
Y\left(W \# \ell\left(S^{1} \times S^{3}\right), \partial W\right)=\frac{1}{\sqrt{2}} Y(N)=-8 \sqrt{2} \pi \sqrt{\left(k_{1}-1\right)\left(k_{2}-1\right)}<0 .
$$

Proof. The above manifold $N$ is a minimal complex surface of general type, and the metric $\bar{g}$ is an $\iota$-invariant Kähler-Einstein metric with constant negative scalar curvature. Hence $\bar{g}$ is a Yamabe metric and $\bar{g}$ attains the Yamabe invariant $Y(N)$. It means that $Y_{[\bar{g}]}(N)=Y(N)$, where $Y_{[\bar{g}]}(N)$ stands for the Yamabe constant of $(N,[\bar{g}])$. Then it follows from $[17$, Theorem 2] and the fact that $\left.\bar{g}\right|_{W}$ is a relative Yamabe metric that

$$
\begin{aligned}
Y_{\left[\left.\bar{g}\right|_{W}\right]}(W, \partial W) & =\frac{1}{\sqrt{2}} Y_{[\bar{g}]}(N)=\frac{1}{\sqrt{2}} Y(N)=-4 \pi \sqrt{2 \chi(N)+3 \sigma(N)} \\
& =-8 \sqrt{2} \pi \sqrt{\left(k_{1}-1\right)\left(k_{2}-1\right)}<0 .
\end{aligned}
$$

Then Corollary 5.4 gives

$$
Y(W, \partial W)=\frac{1}{\sqrt{2}} Y(N)
$$

Let $\ell \geq 1$. Then by a modification of the arguments given in [22, Section 2] and [21, Theorem 1], there exists a sequence of $\hat{\imath}$-invariant metrics $\left\{\hat{g}_{j}\right\}_{j=1}^{\infty}$ on $N \# 2 \ell\left(S^{1} \times S^{3}\right)$ such that

$$
\begin{aligned}
-\left(\liminf _{j \rightarrow \infty} \int_{N \# 2 \ell\left(S^{1} \times S^{3}\right)}\left|R_{\hat{g}_{j}}\right|^{2} d \sigma_{\hat{g}_{j}}\right)^{1 / 2} & =Y\left(N \# 2 \ell\left(S^{1} \times S^{3}\right)\right) \\
& =Y(N)<0 .
\end{aligned}
$$


Recall that $\partial W=\operatorname{Fix}(\hat{\iota})$ is totally geodesic in the manifold $N \# 2 \ell\left(S^{1} \times S^{3}\right)$ with respect to each metric $\hat{g}_{j}$, and then we have

$$
\begin{aligned}
Y\left(W \# \ell\left(S^{1} \times S^{3}\right), \partial W\right) & \geq-\left(\liminf _{j \rightarrow \infty} \int_{W \# \ell\left(S^{1} \times S^{3}\right)}\left|R_{\hat{g}_{j}}\right|^{2} d \sigma_{\hat{g}_{j}}\right)^{1 / 2} \\
& =-\frac{1}{\sqrt{2}}\left(\liminf _{j \rightarrow \infty} \int_{N \# 2 \ell\left(S^{1} \times S^{3}\right)}\left|R_{\hat{g}_{j}}\right|^{2} d \sigma_{\hat{g}_{j}}\right)^{1 / 2} .
\end{aligned}
$$

Combining (6.1), (6.2) with Corollary 5.4, we obtain that

$$
Y\left(W \# \ell\left(S^{1} \times S^{3}\right), \partial W\right)=\frac{1}{\sqrt{2}} Y(N)=-8 \sqrt{2} \pi \sqrt{\left(k_{1}-1\right)\left(k_{2}-1\right)}<0 .
$$

This completes the proof of Corollary 6.4.

6.3. One more family of four-manifolds with negative relative Yamabe invariant. Let $M$ be a Mumford's fake projective plane, that is, a closed complex-hyperbolic surface with Betti numbers $b_{1}=0$ and $b_{2}=$ $b_{2}^{+}=1$, see [2, Chapter V]. From the results of [15], it follows that the manifold $X:=M \#(-M)$ has a $S_{p i n}{ }^{c}$-structure with non-trivial SeibergWitten invariant (however $X$ does not have any symplectic structure). By modifying the technique from [16, Theorem 2] combined with the above fact, it is proved in [12] that $Y(X) \leq-12 \sqrt{2} \pi<0$. It follows from [21] that $Y\left(X \# \ell\left(S^{1} \times S^{3}\right)\right)=Y(X)$ for $\ell \geq 1$. With this understood, the following assertion follows from Corollary 5.4.

Corollary 6.5. Let $M$ be a Mumford's fake projective plane and $D^{4} \subset M$ an embedded disk with $S^{3}=\partial D^{4}$. Then

$$
Y\left(\left(M \backslash \operatorname{int}\left(D^{4}\right)\right) \# \ell\left(S^{1} \times S^{3}\right), S^{3}\right) \leq-12 \pi<0 \quad \text { for } \quad \ell \geq 0 .
$$

Remark 15. The authors are grateful to the referee who brought this example to our attention.

\section{Notes on moduli spaces.}

7.1. Moduli space of positive scalar curvature metrics. Let $M$ be a closed manifold admitting a positive scalar curvature metric. Consider the space of psc-metrics

$$
\mathcal{R i e m}^{+}(M)=\left\{g \in \mathcal{R} \operatorname{iem}(M) \mid R_{g}>0\right\} .
$$


It is known that $\operatorname{Riem}^{+}(M)$ has, in general, many connected components, and that its homotopy groups are nontrivial. For simplicity we assume that $M$ is an oriented manifold. We denote by $\operatorname{Diff}_{+}(M)$ the group of diffeomorphisms preserving the orientation. Then the group $\operatorname{Diff}_{+}(M)$ naturally acts on the space of metrics by pulling back a metric via a diffeomorphism. Clearly this action preserves the space $\operatorname{Riem}^{+}(M)$. Then the moduli space of psc-metrics is defined as $\mathcal{M}^{+}(M)=\mathcal{R i e m}^{+}(M) / \operatorname{Diff}_{+}(M)$.

It is very challenging problem to describe (in any reasonable terms) the topology of the moduli space $\mathcal{M}^{+}(M)$. We suggest here to give an alternative model of the moduli space of psc-metrics. First, we start with the space $\mathcal{C}^{+}(M)$ of positive conformal classes. There is a canonical projection map $p: \operatorname{Riem}^{+}(M) \longrightarrow \mathcal{C}^{+}(M)$, which sends a metric $g$ to its conformal class $[g]$. We prove the following fact.

Theorem 7.1. Let $M$ be a closed manifold of $\operatorname{dim} M \geq 3$. Then the canonical projection map $p: \mathcal{R i e m}^{+}(M) \longrightarrow \mathcal{C}^{+}(M)$ is weak homotopy equivalence.

Proof. We start with the following easy observation.

Lemma 7.2. Let $C \in \mathcal{C}^{+}(M)$, and let $g_{0}, g_{1} \in C$ be psc-metrics. Then $g_{0}$ and $g_{1}$ are psc-homotopic, i.e. there exists a smooth family $\{g(t)\}_{t \in[0,1]} \in C$ of psc-metrics with $g(0)=g_{0}, g(1)=g_{1}$.

Proof. Indeed, we have that $R_{g_{0}}>0$, and $g_{1}=u^{\frac{4}{n-2}} g_{0}$ for $u \in C_{+}^{\infty}(M)$ with the scalar curvature

$$
R_{g_{1}}=u^{-\frac{n+2}{n-2}}\left(-\frac{4(n-1)}{(n-2)} \Delta u+R_{g_{0}} u\right)>0
$$

Then the curve of metrics $g(t)=u(t)^{\frac{4}{n-2}} g_{0} \in C$ with $u(t)=u t+(1-t)>0$ satisfies

$$
\begin{aligned}
R_{g(t)} & =u(t)^{-\frac{n+2}{n-2}}\left(-\frac{4(n-1)}{n-2} \Delta u(t)+R_{g_{0}} u(t)\right) \\
& =u(t)^{-\frac{n+2}{n-2}}\left(t\left[-\frac{4(n-1)}{n-2} \Delta u+R_{g_{0}} u\right]+(1-t) R_{g_{0}}\right) \\
& =u(t)^{-\frac{n+2}{n-2}}\left(t R_{g_{1}} u^{\frac{n+2}{n-2}}+(1-t) R_{g_{0}}\right)>0
\end{aligned}
$$

since both functions $R_{g_{1}} u^{\frac{n+2}{n-2}}$ and $R_{g_{0}}$ are positive. 
Now let $P(C)=\left\{g \in C \mid R_{g}>0\right\}$. Clearly $C \cong C_{+}^{\infty}(M)$ is a convex set. One can easily modify Lemma 7.2 to prove the following assertion.

Lemma 7.3. The subset $P(C) \subset C$ is a convex and contractible set.

Then we notice that both spaces $\operatorname{Riem}^{+}(M)$ and $\mathcal{C}^{+}(M)$ have homotopy types of $C W$-complexes. Thus we can assume (up to homotopy equivalence) that $p: \mathcal{R i e m}^{+}(M) \longrightarrow \mathcal{C}^{+}(M)$ is a fibration. Since $p^{-1}(C)$ is contractible for any conformal class $C$, we obtain that $p$ induces isomorphism in homotopy groups $p_{*}: \pi_{k}\left(\mathcal{R i e m}^{+}(M)\right) \cong \pi_{k}\left(\mathcal{C}^{+}(M)\right)$.

Thus in the homotopy category one does not loose any information by replacing the space $\mathcal{R i e m}^{+}(M)$ by the space of positive conformal classes $\mathcal{C}^{+}(M)$.

The space $\mathcal{C}(M)$ is the orbit space of the action (left multiplication) of the group $C_{+}^{\infty}(M)$ on the space of metrics $\operatorname{Riem}(M)$. It is convenient to refine this construction (as it is done in [19]) for manifolds with a base point. Let $x_{0} \in M$ be a base point. We consider the following subspace of $C_{+}^{\infty}(M)$ :

$$
C_{+, x_{0}}^{\infty}(M)=\left\{u \in C_{+}^{\infty}(M) \mid u\left(x_{0}\right)=1\right\} .
$$

We denote by $\mathcal{C}_{x_{0}}(M)$ the orbit space of the induced (left) action of $C_{+, x_{0}}^{\infty}(M)$ on $\operatorname{Riem}(M)$. There is a canonical map $p_{1}: \mathcal{C}_{x_{0}}(M) \longrightarrow \mathcal{C}(M)$ which is a homotopy equivalence since $p_{1}^{-1}(C) \cong \mathbf{R}$. Let $\mathcal{C}_{x_{0}}^{+}(M)=p_{1}^{-1}\left(\mathcal{C}^{+}(M)\right)$. To construct an appropriate moduli space, we assume that $M$ is a connected manifold, and consider the following subgroup of the diffeomorphism group $\operatorname{Diff}_{+}(M)$ :

$\operatorname{Diff}_{x_{0},+}(M)=\left\{\phi \in \operatorname{Diff}_{+}(M) \mid \phi\left(x_{0}\right)=x_{0}, \quad d \phi_{x_{0}}=I d: T M_{x_{0}} \rightarrow T M_{x_{0}}\right\}$.

The group Diff $x_{x_{0},+}(M)$ inherits the action on the spaces $\mathcal{C}(M)$ and $\mathcal{C}_{x_{0}}(M)$. It turns out that this action is free for all manifolds except the sphere $S^{n}$.

Theorem 7.4. Let $M$ be a closed, connected oriented manifold of $\operatorname{dim} M=$ $n \geq 3$, and $x_{0} \in M$.

(1) If $M$ is not diffeomorphic to the sphere $S^{n}$, then the group $\operatorname{Diff}_{x_{0},+}(M)$ acts freely on the space $\mathcal{C}_{x_{0}}(M)$.

(2) If $M=S^{n}$, then the group $\operatorname{Diff}_{x_{0},+}(M)$ acts freely on the space $\mathcal{C}_{x_{0}}\left(S^{n}\right) \backslash p_{1}^{-1}\left(C_{\mathrm{can}}\right)$, where $C_{\mathrm{can}}$ is the conformal class of the standard metric on $S^{n}$. Each class $C_{x_{0}} \in p_{1}^{-1}\left(C_{\text {can }}\right)$ has an isotropy group $\mathcal{H}_{C_{x_{0}}} \cong \mathbf{R}^{n}$ (here $\mathbf{R}^{n}$ is the group of parallel translations of $\left.\mathbf{R}^{n} \cong S^{n} \backslash\left\{x_{0}\right\}\right)$. 
Proof. Let $(M, C) \not\left(S^{n}, C_{\text {can }}\right)$ and $C_{x_{0}} \in p_{1}^{-1}(C)$. Assume that $\phi^{*}\left(C_{x_{0}}\right)=$ $C_{x_{0}}$. It follows from [20] and [18] that there exists a metric $g \in C_{x_{0}}$ such that $\phi^{*} g=g$, i.e. $\phi$ is an isometry of $(M, g)$. Then the conditions $\phi\left(x_{0}\right)=x_{0}$, $d \phi_{x_{0}}=I d$ imply that $\phi=I d$.

Let $(M, C) \cong\left(S^{n}, C_{\text {can }}\right)$ and $C_{x_{0}} \in p_{1}^{-1}\left(C_{\text {can }}\right)$. We choose a coordinate system on $S^{n} \cong \mathbf{R}^{n} \cup\{\infty\}$ via the stereographic projection from $x_{0}$ such that $x_{0}=\infty$. Let $\phi^{*}\left(C_{x_{0}}\right)=C_{x_{0}}$. Then the conditions $\phi\left(x_{0}\right)=x_{0}, d \phi_{x_{0}}=I d$ imply that $\phi(x)=x+b$, where $b \in \mathbf{R}^{n}$.

Remark 16. We are grateful to the referee for pointing out that the above action is not free for the standard sphere.

Clearly the space $\mathcal{C}_{x_{0}}^{+}(M)$ of positive conformal classes is invariant under this action. We define the moduli space $\mathcal{M}_{x_{0}, \text { conf }}^{+}(M)$ of positive conformal structures as the orbit space of the action of $\operatorname{Diff}_{x_{0},+}(M)$ on $\mathcal{C}_{x_{0}}^{+}(M)$. Let $M$ be not diffeomorphic to the sphere $S^{n}$. Then we have the following diagram of Serre fiber bundles

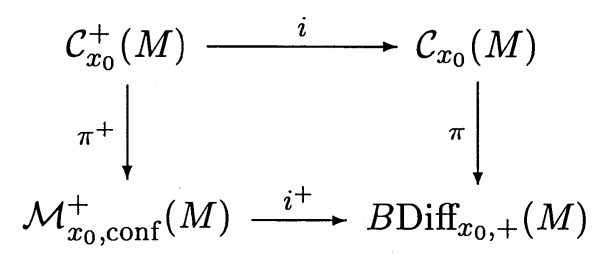

Here $B \operatorname{Diff}_{x_{0},+}(M)$ is the classifying space of the group Diff $x_{x_{0},+}(M)$, which we identify with the orbit space $\mathcal{C}_{x_{0}}(M) / \operatorname{Diff}_{x_{0},+}(M)$ (since the action is free, and the space $\mathcal{C}_{x_{0}}(M)$ is contractible).

Remark 17. In the case $M=S^{n}$, the action $\operatorname{Diff}_{x_{0},+}\left(S^{n}\right)$ on $\mathcal{C}_{x_{0}}^{+}\left(S^{n}\right)$ is not free. However the orbit space has the same homotopy type as the classifying space $B \operatorname{Diff}_{x_{0},+}\left(S^{n}\right)$ since the isotropy groups are contractible.

We address the following problem.

Problem 1. What is the rational homotopy type of the space $\mathcal{M}_{x_{0}, \text { conf }}^{+}(M)$ ?

7.2. Conformal isotopy and concordance. It is well-known that isotopic psc-metrics are concordant, see [9] and [8]. It is still not known if the converse is true for $\operatorname{dim} M \geq 5$. (Recently, Ruberman [24] proved that, in the 4-dimensional case, concordance of psc-metrics does not imply isotopy of such metrics.) We would like to address the "conformal analogue" of this problem. 
Let $C_{0}, C_{1} \in \mathcal{C}^{+}(M)$ be two positive conformal classes. One defines an isotopy of positive conformal classes in the obvious way. We say that the conformal classes $C_{0}$ and $C_{1}$ are conformally concordant if

$$
Y\left(M \times[0,1], M \times\{0,1\} ; C_{0} \sqcup C_{1}\right)>0 .
$$

Theorem 5.1 implies the following result:

Corollary 7.5. Conformal concordance is an equivalence relation on $\mathcal{C}^{+}(M)$.

We would like to spell out the following conjecture:

Conjecture 7.6. Let $M$ be a closed manifold of $\operatorname{dim} M \geq 5$ admitting a psc-metric. If $C_{0}, C_{1} \in \mathcal{C}^{+}(M)$ are conformally concordant, then the classes $C_{0}, C_{1}$ are isotopic in $\mathcal{C}^{+}(M)$.

7.3. Conformal cobordism. Two manifolds $\left(M_{0}, g_{0}\right),\left(M_{1}, g_{1}\right)$ with psc-metrics $g_{0}, g_{1}$ are said to be psc-cobordant if there exists a compact manifold $(W, \bar{g})$ with $\partial W=M_{0} \sqcup\left(-M_{1}\right)$ and a psc-metric $\bar{g}$ such that:

$$
\left.\bar{g}\right|_{M_{j}}=g_{j}, \quad j=1,2, \quad \text { and } \bar{g}=g_{j}+d r^{2} \text { near } M_{j} .
$$

These psc-cobordism groups have been studied in [4], [8], [11], [23], [26].

We define the conformal analogue of the psc-cobordism relation in terms of the relative Yamabe invariant. Let $\left(M_{0}, C_{0}\right),\left(M_{1}, C_{1}\right)$ be two closed manifolds equipped with positive conformal classes. We call such manifolds positive conformal manifolds. Then $\left(M_{0}, C_{0}\right),\left(M_{1}, C_{1}\right)$ are conformally cobordant if there exists a compact manifold $W$ with $\partial W=M_{0} \sqcup\left(-M_{1}\right)$ such that the relative Yamabe invariant

$$
Y\left(W, M_{0} \sqcup\left(-M_{1}\right) ; C_{0} \sqcup C_{1}\right)>0 .
$$

Theorem 5.1 also implies the following result:

Corollary 7.7. Conformal cobordism is an equivalence relation on the category of positive conformal manifolds.

Remark 18. The definition of the conformal cobordism may be essentially refined in the way suggested by S. Stolz [26]. This leads to the conformal cobordism groups. We have studied these cobordism groups in [1]. 


\section{References.}

[1] K. Akutagawa, B. Botvinnik, Manifolds of positive scalar curvature and conformal cobordism theory, to appear in Math. Ann.

[2] W. Barth, C. Peters, A. Van de Ven, Compact complex surfaces, Springer-Verlag, Berlin, 1984.

[3] A. Besse, Einstein manifolds, Springer, Berlin, 1987.

[4] B. Botvinnik, P. Gilkey, The eta invariant and metrics of positive scalar curvature, Math. Ann. 302 (1995), 507-517.

[5] I. Chavel, Eigenvalues in Riemannian geometry, Pure and App. Math. 115, Academic Press, 1984.

[6] P. Cherrier, Problèmes de Neumann non linéaires sur les variétés riemanniennes, J. Funct. Anal. 57 (1984), 154-206.

[7] J. Escobar, The Yamabe problem on manifolds with boundary, J. Diff. Geom. 35 (1992), 21-84.

[8] P. Gajer, Concordances of metrics of positive scalar curvature, Pacific J. Math. 157 (1993), 257-268

[9] M. Gromov, H. B. Lawson, The classification of simply connected manifolds of positive scalar curvature, Ann. of Math. 111 (1980), 423-434.

[10] M. Gromov, H. B. Lawson, Positive scalar curvature and the Dirac operator on complete Riemannian manifolds, Publ. Math. Inst. Hautes Études Sci. No. 58, (1983), 83-196 (1984).

[11] B. Hajduk, On the obstruction group to existence of Riemannian metrics of positive scalar curvature, Global differential geometry and global analysis (Berlin, 1990), 62-72, Lecture Notes in Math. 1481, SpringerVerlag, 1991.

[12] M. Ishida, Seiberg-Witten invariants and genericity of four-manifolds without Einstein metrics, preprint.

[13] O. Kobayashi, Scalar curvature of a metric with unit volume, Math. Ann. 279 (1987), 253-265.

[14] S. Kobayashi, Transformation groups in differential geometry, SpringerVerlag, 1972. 
[15] D. Kotschick, J. Morgan, C. Taubes, Four-manifolds without symplectic structures but with nontrivial Seiberg-Witten invariants, Math. Res. Lett. 2 (1995), 119-124.

[16] C. LeBrun, Four-manifolds without Einstein metrics, Math. Res. Lett. 3 (1996), 133-147.

[17] C. LeBrun, Kodaira dimension and the Yamabe invariant, Comm. Anal. Geom. 7 (1999), 133-156.

[18] J. Lelong-Ferrand, Geometrical interpretations of scalar curvature and regularity of conformal homeomorphisms, Differential geometry and relativity, 91-105, Math. Phys. and Appl. Math., Vol. 3, Reidel, Dordrecht, 1976 .

[19] J. Morava, H. Tamanoi, A vanishing theorem for the conformal anomaly in dimension > 2, Proc. Amer. Math. Soc. 100 (1987), 767-774.

[20] M. Obata, The conjectures on conformal transformations of Riemannian manifolds, J. Diff. Geom. 6 (1971), 247-258.

[21] J. Petean, Computation of the Yamabe invariant, Math. Res. Lett. 5 (1998), 703-709.

[22] J. Petean, G. Yun, Surgery and the Yamabe invariant, Geom. Funct. Anal. 9 (1999), 1189-1199.

[23] J. Rosenberg, S. Stolz, Metric of positive scalar curvature and connection with surgery, Surveys on surgery theory, Vol 2, 353-386, Ann. of Math. Studies 149, Princeton Univ. Press, 2001.

[24] D. Ruberman, Positive scalar curvature, diffeomorphisms and the Seiberg-Witten invariants, Geom. and Topol. 5 (2001), 895-924.

[25] R. Schoen, Variational theory for the total scalar curvature functional for Riemannian metrics and related topics, Topics in calculus of variations (Montecatini Terme, 1987), 120-154, Lecture Notes in Math. 1365, Springer-Verlag, 1989.

[26] S. Stolz, Concordance classes of positive scalar curvature metrics, to appear.

[27] C. Taubes, The Seiberg-Witten invariants and symplectic forms, Math. Res. Lett. 1 (1994), 809-822. 
Department of Mathematics

SHIZUOKA UNIVERSITY

ShIZUOKA 422-8529, JAPAN

E-mail: smkacta@ipc.shizuoka.ac.jp

Department of Mathematics

UNIVERSITY OF OREGON

EUGENE, OR 97405, USA

E-mail: botvinn@math.uoregon.edu 